\title{
Zebrafish Wnt9b Patterns the First Pharyngeal Arch into D-I-V Domains and Promotes Anterior-Medial Outgrowth
}

\author{
Henry Wells Jackson1, Divakar Prakash², Mark Litaker³, Tracie Ferreira4, \\ Peter A. Jezewski ${ }^{*}$ \\ ${ }^{1}$ Department of Genetics, University of Alabama at Birmingham, Birmingham, USA \\ ${ }^{2}$ University of Alabama at Birmingham School of Dentistry, Institute of Oral Health Research, Birmingham, USA \\ ${ }^{3}$ Department of Clinical and Community Sciences, University of Alabama at Birmingham School of Dentistry, \\ Birmingham, USA \\ ${ }^{4}$ Department of Bioengineering, University of Massachusetts Dartmouth, Dartmouth, MA, USA \\ ${ }^{5}$ Department of Pediatric Dentistry, University of Alabama at Birmingham School of Dentistry, \\ Birmingham, AL, USA \\ Email: peterj@uab.edu
}

Received 21 May 2015; accepted 3 July 2015; published 6 July 2015

Copyright (C) 2015 by authors and Scientific Research Publishing Inc. This work is licensed under the Creative Commons Attribution International License (CC BY). http://creativecommons.org/licenses/by/4.0/

(c) (†) Open Access

\section{Abstract}

Disrupted morphogenesis and growth of the embryonic maxillary jaw lead to oral facial clefting in humans (OFC) and result in an incompletely formed secondary mouth and face. A requirement for Wnt signaling and Wnt9b in particular are postulated in the etiology of OFC from association studies in humans and from animal models. Loss of murine Wnt9b leads to reduced upper jaw (maxillary) outgrowth and OFC, though the signaling architecture leading to this phenotype is poorly understood. Previous murine Wnt9b studies largely overlooked cranial neural crest cell (CNCC) patterning events and instead focused on later events during fusion of facial prominences. Using zebrafish and a morpholino-mediated knockdown approach, we demonstrate functional requirements for Wnt9b signaling during two crucial stages of facial development: 1) CNCC patterning into Dorsal-Intermediate-Ventral (D-I-V) domains; and 2) facial outgrowth during the primary to secondary mouth transition (PM to SM). Zebrafish embryos deficient for Wnt9b (Wnt9b morphants) exhibit an open bite with fused jaw joints as well as a flat face. Open bite and jaw joint fusion in Wnt9b morphants phenocopies characteristics of edn1 pathway is mutant zebrafish with disrupted D-I-V patterning of CNCC. Expression studies show Wnt9b morphants exhibit perturbed expression of edn1 signaling targets including $d \mathrm{~lx}_{2} a$, jag $1 \mathrm{~b}$, and msxe, consistent with disrupted CNCC patterning. Wnt9b morphant upper jaws have stunted outgrowth reminiscent of murine Wnt9b mutants and Wnt9b morphant skulls phenocopy the broad class of foreshortened skull zebrafish mutants known as hammerheads. Wnt9b morphants show upregulated expression of

\footnotetext{
"Corresponding author.
}

How to cite this paper: Jackson, H.W., Prakash, D., Litaker, M., Ferreira, T. and Jezewski, P.A. (2015) Zebrafish Wnt9b Patterns the First Pharyngeal Arch into D-I-V Domains and Promotes Anterior-Medial Outgrowth. American Journal of Molecular Biology, 5, 57-83. http://dx.doi.org/10.4236/ajmb.2015.53006 
pitx $2 a$ after the opening of the primary mouth and disrupted expression of Wnt5b which is consistent with disrupted chondrocyte stacking. Strong upregulation of dorsal mesodermal frzb expression in the prechordal plate of Wnt9b morphants suggests a role for Wnt9b in primary mouth induction or maintenance. Collectively these results argue that Wnt9b has a much earlier developmental requirement. This work draws attention to potential vertebrate homologies that pattern CNCC and facial outgrowth and therefore calls for a reexamination of Wnt9b's role during mammalian craniofacial development.

\title{
Keywords
}

\author{
Cleft, Morphogenesis, Patterning, Cranial Neural Crest Cell, Pharyngeal Arch, Primary/Secondary \\ Mouth
}

\section{Introduction}

The Genetics of OFC and Relevance to Human Disease

Oral-facial clefting (OFC) of the upper jaw (maxilla) is a common, severe, and costly birth disorder affecting between 1/500 and 2/1000 live births per year, depending on population [1] [2]. OFC patients lack the appropriate growth and morphogenesis of the upper jaw. Patients with OFC may undergo treatment involving substantial medical intervention, with severe OFC requiring several expensive surgeries over a number of years to restore adequate oral function [3]. In light of the high incidence and overbearing costs of treatment, a better understanding of pathogenic mechanisms, both early and late, becomes an imperative that will lead to more focused preventive and treatment strategies.

OFC can be either syndromic (30\% of cases; generally with severe comorbidities) or non-syndromic (NSOFC) (70\% of cases), and both types have a definite genetic etiology [4]-[7]. Currently over 400 Mendelian syndromes include OFC as a characteristic feature, including OFC with Tooth Agenesis (MSX1), X-linked Ankyloglossia and Cleft Palate (TBX22), Tetra Amelia (Wnt3) and Robinow Syndrome (Wnt5a) [8]-[13]. While syndromic forms are more severe, they are fortunately rare Mendelian disorders. NS-OFC, on the other hand, is more common and represents a considerable proportion of morbid human birth disorders [14]. Unlike syndromic OFC, NS-OFC is considered as a polygenic, complex, and multifactorial disorder [15]-[17]. Several association studies have linked Wnts, including Wnt9b, to human OFC [18] [19].

Zebrafish as a Proxy to Study Mammalian OFC

Zebrafish as a model vertebrate has been proven to be invaluable for insight into human disorders. However, it is important in the present context that the homologies and differences that underlie the developmental processes and adult jaw structures in both fish and mammals are understood.

The vertebrate skull can be divided into three components: the chondrocranium, the splanchnocranium, and the dermatocranium [20]-[22]. Vertebrate jaws are composed of differential contributions of the latter two components in fish and mammals. The splanchnocranium is endochondral bone found originally within the pharyngeal arch bars whereas the dermatocranium is membrane bone formed without a cartilage precursor.

The anterior-most pharyngeal arch (first PA) endochondral as well as multiple membrane bones both contribute to the upper and lower jaws in zebrafish [21]. The endochondral contribution to the embryonic zebrafish lower jaw is the Meckel's cartilage (MK) while the upper jaw consists of two laterally paired palatoquadrates (PQs) and the single ethmoid palate (ETH) midline element [23] [24]. Membrane bones, like the maxillary and premaxillary of the upper jaw and the dentary in the lower jaw, represent some of the membrane components of the fish jaws. However, in the tetrapod lineages towards current day mammals, the first pharyngeal arch endochondral components largely evolved away from jaw functions [21]. In mammals the palatoquadrate contributes to the incus within the middle ear and to the alisphenoid portion or greater wing of the sphenoid bone. Therefore in mammals the dermal bones contribute to a much greater extent to the upper and lower jaws when compared to zebrafish. Further, while homologous structural components (e.g. the palatoquadrate) of the first pharyngeal arch are found in different parts of the fish or mammalian skull, the jaws themselves are functionally homologous and their formation may be regulated by conserved gene regulatory networks [25]. Thus for the present study, while the MK and PQ cartilages are not structurally homologous to the dermal mammalian jaw bones, they can 
be considered as appropriate proxies for studying the genetic mechanisms that control vertebrate jaw formation through potentially homologous patterning mechanisms [21].

Wnt Growth Factor Signaling

The Wnt family is an evolutionarily conserved group of extracellular glycoprotein ligands comprising 19 genes in humans [26]. Wnt signaling is complex, and depending on tissue-specific ligand/receptor/coreceptor/ inhibitor context, Wnt signaling follows either $\beta$-catenin dependent canonical or $\beta$-catenin independent noncanonical pathways (C-Wnt versus NC-Wnt, respectively) [27]-[29]. C-Wnt signals localize $\beta$-catenin to the nucleus. The NC-Wnt pathway comprises a set of overlapping pathways that include conferring migratory properties to cells such as convergent extension, in addition to general antagonism of C-Wnt signals [30] [31]. Mutations in Wnts that signal through both canonical and noncanonical pathways can cause OFC and are implicated within animal models of craniofacial development. C-Wnt studies revealed differential requirements for surface epithelial $\beta$-catenin in upper or lower jaw development [32]. Moreover, studies of NC-Wnt demonstrate that Wnt5a null mice have foreshortened snouts and a complete cleft secondary palate [33] [34]. Interestingly, the zebrafish pipetail mutant harbors a null Wnt5b allele and is a member of a broader class of zebrafish hammerhead mutants, all of which display severe anterior-posterior foreshortening of the face and jaw elements [35] [36]. The fact that loss of Wnt5's disrupts facial outgrowth in humans, mice, and zebrafish argues for Wnt5's participation in homologous developmental pathways regarding vertebrate faces and jaws [12]. Therefore, multiple lines of evidence point to a conserved role for Wnt signaling within vertebrate lineages, wherein loss of either C-Wnt or NC-Wnt may lead to an OFC phenotype.

Association studies have implicated WNT9s in OFC [18] [19]. Regarding Wnt9b in animal models, A/WySn mice harbor a hypomorphic Wnt9b allele and demonstrate partially penetrant NS-OFC (around 20\% of pups effected) while full knockout of Wnt9b increases penetrance of OFC to over 90\% [37]. Both A/WySn and Wnt9b knockout mice demonstrate quantitative maxillary growth deficiencies, not unlike snout foreshortening in Wnt5a mutants [33]. Murine Wnt9b is expressed in the medial and lateral nasal prominences as well as in the epithelia of the first pharyngeal arch derived maxillary prominence [38]. Furthermore, Wnt9b was suggested to signal via $\beta$-catenin in a murine study of the events surrounding the late fusion of facial prominences that form the secondary mouth [39]. A concurrent study of these late fusion events in a mouse model demonstrated that Wnt9b was part of a conserved Pbx-p63-Irf6 regulatory module that promoted epithelial apoptosis, which otherwise lead to highly penetrant cleft lip and palate [40].

In zebrafish, we and others showed that Wnt9b is expressed maternally and during early zygotic phases [41]-[43]. Later, from about one day post fertilization (dpf) onwards, Wnt9b is expressed within the first pharyngeal arch (PA) ectodermal epithelia that overlies the arch's cranial neural crest cells (CNCCs), which have mostly finished their migration into the ventral half of the embryo by 24 hours post fertilization (hpf) [41]. Robust expression of Wnt9b in the first arch ectoderm persists past 40hpf, surrounding the primary mouth as it initially opens at 38 - 40hpf [44]. Zebrafish Wnt9b facial expression (from 24 - 40 hours post fertilization) is remarkably similar to murine Wnt9b facial expression (from E9.5-11) at developmentally homologous timepoints, suggesting Wnt9b may have an integral and conserved role in vertebrate facial development across myriad taxa [38] [43]. Importantly, Wnt9b expression coincides with other signals from the pharyngeal ectodermal epithelia including the well-studied edn1 pathway (described below), so Wnt9b expression is therefore well-placed spatially and temporally to influence CNCC [45].

$D$-V to D-I-V Patterning: edn1, dlx2a, and sox9a

The vertebrate jaws are composed of nerves, muscles, bones and cartilages, all of which are derived from the CNCC mesenchyme of the pharyngeal arches [46]. Vertebrate jaws develop from early dorsal/maxillary, intermediate/joint and ventral/mandible divisions within the CNCCs (D-I-V domains) [25] [47]. These three jaw domains arise from within the first PA. In zebrafish at 1dpf, PA1 has an initial D-V division such that the V domain is refined into I-V subdomains by 1.5dpf through the well-studied edn1 signaling pathway [45] [48]-[50]. It is important to note that the Edn1 pathway is highly conserved in vertebrates, as Edn1 mutant mice and edn1 mutant zebrafish share disruption of both jaw elements and genetic markers, described below. edn 1 mutant zebrafish have an open bite and fused jaw joint, defects confined to intermediate and ventral domain jaw cartilage elements. Expression studies show edn1 is required for expression of intermediate domain genes msxe and bapx1, as well as ventral hand2 [45] [50]. edn1 signaling not only promotes intermediate and ventral but also represses dorsal genes like jag $1 b$, a dorsal marker that is expanded in the absence of edn 1 [51] [52]. edn1 signaling defines the three jaw domains by regulating expression of a nested bigene Dlx code which is also highly conserved across vertebrates. In this model, $d l x 1 a / 2 a$ are expressed throughout all three domains, with $d l x 5 a / 6 a$ 
and $d l x 3 b / 4 b$ expressed in progressively more discrete intermediate and ventral domains respectively [48] [53]. Loss of specific Dlxs frequently cause jaw defects spatially confined to the respective Dlx's expression domain. For example edn 1 mutants show loss of intermediate $d l x 5 a / 6 a$ and $d l x 3 b$ while $d l x 2 a$ expression is absent only from the ventral most portions of PA1 [45]. Jaw cartilage defects in edn1 mutants (confined to intermediate and ventral domains) are therefore correlated with specific Dlx expression domains. It is curious that dorsal $d l x 2 a$ expression does not appear to rely on edn1, suggesting dorsal $d l x 2 a$ 's activation by another factor. Yet zebrafish studies specifically targeting $d l x 2 a$ showed disruption of multiple cartilages including the upper jaw, as well as perturbed expression of the chondrocyte marker sox9a [54]. Moreover, Dlx2 null mice show skull dermal bone loss and abnormalities that result in cleft palate of the upper jaw, further suggesting a homologous role for $D l x 2$ and $d l x 2 a$ in facial formation [55] [56]. Taken together, this evidence strongly suggests the existence of a conserved gene-regulatory network among vertebrates, including edn1 that patterns CNCC of the pharyngeal arches into the face and jaws.

Primary and Secondary Mouths

Initiation of the primary mouth, a deeply conserved process within vertebrates, involves direct contact of oral ectoderm with the foregut endoderm [57] [58]. The oral ectoderm, which is the presumptive primary mouth, is specified very early and develops from the most anterior portion of a horseshoe shaped preplacodal ectoderm (PPE) [59] [60]. The preplacodal ectoderm region subsequently divides into multiple subdomains, becoming the single oral, and the bilateral nasal, optic, and otic cranial sensory placodes [61]. All the cranial placodes share common early pathway genes, including Six and Eya homologs, while only the oral placode requires Pitx gene expression for its specification, making it an ideal marker for the primary mouth [57] [62] [63]. Oral placodal competence requires this region be protected from caudalizing signals including Wnts and Bmps. The prechordal plate mesoderm secretes the anti-Wnt factor frzb that may help protect the oral placode from such caudalizing signals. For example, it has already been shown that overstimulation of C-Wnt or loss of anti-Wnt signals, including frzb, severely reduces the primary mouth in Xenopus [64].

The secondary mouth subsequently arises from anteriorly directed outgrowth of the tissues surrounding to the primary mouth, including the D-I-V patterned CNCC of the pharyngeal arches. During the transistion from the primary to the secondary mouth, these tissues grow anteriorly from around the primary mouth and eventually form the jaws, the face and the secondary mouth. Also during secondary mouth outgrowth, the primary mouth will fully open a communication channel with the foregut through dissolution of the buccopharyngeal membrane [57]. Interestingly, some extreme cases of human OFC can result from combinatorial failure of primary mouth opening as well as perturbed secondary mouth outgrowth, suggesting a common genetic mechanism that may govern both tissues [65]. However, very little is known regarding how the first pharyngeal arch primordia interact with the primary mouth and how these tissues subsequently transition into forming the jaws and the oral cavity of the secondary mouth. Given the precedents of human OFC with disruption of one or both mouth structures, further investigation of the primary to secondary mouth transition will likely reveal promising new insights into OFC pathogenesis.

We present a Wnt9b deficient phenotype in zebrafish as a relevant disease model to understanding human OFC disorders. Wnt9b is required for 1) D-I-V patterning of CNCC and 2) outgrowth of the secondary mouth. Wnt9b morphants demonstrate jaw malformations indicative of edn1 pathway mutants and hammerhead class mutants. Our expression analyses confirm that Wnt9b operates upstream of the edn1 pathway to pattern the CNCC. At later timepoints during jaw cartilage condensation and outgrowth, Wnt9b deficiency causes disrupted expression of Wnt5b, which often plays a role in convergent extension processes, as well as the chondrocyte differentiation marker sox $9 a$, consistent with disrupted jaw cartilage outgrowth. Finally, we provide evidence that Wnt9b opposes the anti-Wnt signal frzb in the prechordal plate, and this repulsion may influence downstream primary mouth induction and maintenance.

Our results demonstrate a complex requirement for much earlier Wnt9b influence upon normal facial development beyond the scope of simply controlling the final stages of proper fusion of facial prominences. These findings bring into question the etiopathology of OFC. If these findings were to be replicated within mammals they would redefine the timeline of cleft susceptibility and show that genetic disruption far preceding the cleft may be causal.

\section{Methodologies}

Zebrafish Husbandry and Maintenance 
This study was carried out in strict accordance with the recommendations provided by the University of Alabama Institutional Animal Care and Use Committee (approved protocol number: 120909436). Breeding adult zebrafish of $A B$ background were provided by the UAB Nutrition Obesity Research Center Aquatic Animal Research Core and adults were maintained on a reference diet described previously and at 27.5 degrees Celsius and a light cycle of 14 hours light and 10 hours dark [66]. Embryos were collected from multiple breedings of pairs of adults. All embryos for this study were aged in fish system water in a light cycle incubator (14 hours light, 10 hours dark) maintained at 28.5 degrees Celsius. Developmental staging of embryos was determined from hours post fertilization (hpf) or days post fertilization (dpf).

\section{Morpholino Preparation and Microinjections}

Translation blocking and splice blocking morpholinos (TB-MOs or SB-MOs, respectively) against Wnt9b were designed by Gene Tools, LLC. The MO sequences used in this study were as follows: Wnt9b-TB-MO (TBMO), CAGTCCTCGGAAGCCCGGTGCACAT. Wnt9b-SB-MO (SB-MO), ACCTGTAAGCCTAACGAAAACACAA. Wnt9b-SB-MM (SB-MM), CCTcTAAcCCTAAgGAAtAgACAA. Morpholinos arrived as 300 nmols of lyophilized powder. Morpholinos were resuspended in $300 \mathrm{uL}$ of molecular grade water to make $1 \mathrm{mM}$ stocks according to manufacturer's instructions. Concentrations of morpholinos were determined using a spectrophotometer. $1 \mathrm{mM}$ stocks are stored in the dark at room temperature. Prior to embryo injections, morpholinos were diluted in injection solution consisting of molecular grade water with $12.5 \%$ phenol red dye, which serves as an innocuous indicator to assess morpholino delivery into embryos. In preparation for injections, needles were calibrated [67]. 2 ul morpholino solution was back-loaded into a needle using a micropipetter, and the tip of the needle was broken using ultrafine forceps. The pico injector was set to an initial injection pressure of 8.5 $\mathrm{lb} / \mathrm{in}^{2}$ for 30 msec pulse. Injection volume was calculated by injecting solution into a 1-lambda microcapillary (Drum- mond Scientific). 10 injection pulses were delivered into the capillary, and the volume of injected liquid was measured using a ruler viewed under a dissecting microscope. $1 \mathrm{~mm}$ length represents a volume of $30 \mathrm{nl}$. The injection pressure and time of the pulse was then adjusted to accurately and precisely deliver $3 \mathrm{nl}$ of morpholino per injection pulse, or $1 \mathrm{~mm}$ length of liquid in 10 injection pulses. Dilutions of morpholino were calibrated so that $3 \mathrm{nl}$ of morpholino contained the exact ng amount described in the study, and all injected embryos received $3 \mathrm{nl}$ of morpholino.

Synthetic mRNA was generated from linearized Wnt9b plasmid used in our previous publication [41] and the mMessage mMachine T7 ULTRA Transcription Kit (Ambion). The concentration of purified Wnt9b mRNA was determined using a spectrophotometer and the mRNA was diluted in nuclease-free water with $12.5 \%$ phenol red dye to deliver either $140 \mathrm{pg}$ or $280 \mathrm{pg}$ in a $3 \mathrm{nl}$ injection pulse.

Alcian Blue Staining

Alcian blue staining was performed on test and control embryos to visualize jaw cartilage elements in 5dpf aged embryos. Prior to alcian blue staining, embryos were injected at the 1 cell stage and aged to $5 \mathrm{dpf}$ as described. At 5dpf, embryos were photographed, euthanized, and fixed in $4 \%$ paraformaldehyde/PBS solution overnight at 4 degrees. Embryos were then dehydrated in 100\% ethanol overnight and stored until use. Prior to staining, embryos were bleached in hydrogen peroxide solution to remove pigmentation. Alcian blue staining was carried out as described, and stained embryos were cleared through a glycerol/PBT gradient. Cleared embryos were photographed in $80 \%$ glycerol/PBT using brightfield microscopy and Nikon NIS elements software.

Statistical Methods

Whole skull and palatoquadrate length measurement analysis was carried out using a one-way ANOVA, followed with Tukey's HSD for pairwise comparisons of group means. Significance of palatoquadrate cell number and stacked cell sides was calculated using a T-Test comparing mean values of controls and morphants. qRTPCR fold change significance was calculated using a T-Test comparing mean fold changes. Error bars in all figures represent the standard error of means within each group.

\section{Embryo Dissections and Jaw Cartilage Measurements}

Whole skulls or palatoquadrate jaw cartilages were dissected out of $5 \mathrm{dpf}$ alcian blue stained embryos. Whole skulls or individual palatoquadrates were flat mounted in $80 \%$ glycerol/PBT and photographed using brightfield microscopy and Nikon NIS elements software. At least 6 whole skulls per group and at least 10 palatoquadrates per group were measured.

Cell counting and stacked cell side counting was performed according to a previously described assay [24]. Uninjected control embryo palatoquadrates were compared to TB-MO (5.3 ng) injected embryo palatoquadrates. For uninjected controls, values from four pairs of palatoquadrates from four embryos were averaged. For mor- 
phants, values from 10 pairs of palatoquadrates from 10 embryos were averaged.

Reverse Transcriptase PCR (regular RT-PCR)

Uninjected control, SB-MM injected control and SB-MO injected embryos were aged to 24hpf or 28hpf and homogenized using a Trizol kit (Ambion). Total RNA was then purified and $1 \mu \mathrm{g}$ total RNA per group was used with First Strand cDNA synthesis Superscript III kit (Invitrogen) to make cDNA preps. These preps were used as template along with Wnt9b specific primers to detect Wnt9b transcripts: Wnt9b-Forward

5'-GTCATCATGTGCACCGGGCTTC-3', Wnt9b-Reverse 5'-CGCTTTGGCAAGCGCGTGAG-3'. Total cDNA template amounts were standardized among the three groups (uninjected, SB-MM, SB-MO) and equal reaction volumes from the PCR reactions were run in $1 \%$ agarose gels.

Quantitative Reverse Transcriptase PCR ( $q R T-P C R)$

Primer sequences for qRT-PCR are included in the table below. For qRT-PCR experiments, embryos were injected with SB-MM (2.6 ng) control or SB-MO $(2.6 \mathrm{ng})$ morpholinos at the 1 cell stage and aged to $24 \mathrm{hpf}$ or 28hpf as described. For each biological replicate, approximately 50 embryos were homogenized and total RNA extracted using a Trizol kit (Ambion). cDNA was synthesized using $1 \mu \mathrm{g}$ of total RNA and the First Strand cDNA synthesis SuperScript III kit (Invitrogen). $20 \mu \mathrm{l}$ cDNA reactions were diluted to $100 \mu$ in nuclease free water and stored at $-20^{\circ} \mathrm{C}$ until use. All qRT-PCR reactions were run using Applied Biosystems Fast SYBR Green mastermix and an Applied Biosystems 7500 Fast PCR machine. For each gene measured, reactions were run in triplicate and normalized using b2m (Qiagen 330001 PPZ00226A) as a reference gene. We also tested other reference genes, including acta1b (330001 PPZ05295A), ldha (330001 PPZ00298A), rpl13a (330001 PPZ10281A) and $t f r 1 a$ (330001 PPZ07105A) but found $b 2 m$ to be the most stable under our experimental conditions. Fold changes were calculated using the $\Delta \Delta \mathrm{Ct}$ method. Each gene was measured in three technical replicates each of three biological replicates, and fold changes were averaged over the three biological replicates.

\section{Table 1. qRT-PCR and WISH Primers.}

\begin{tabular}{|c|c|c|}
\hline Gene Name - Method & Forward Primer & Reverse Primer \\
\hline axin2 - qRT-PCR & CСTTACATTCGCAGCCGGAGTCTG & TGAGAGCGATCTGTACTGTCAGCTG \\
\hline$d l x 2 a-\mathrm{qRT}-\mathrm{PCR}$ & GCACCTACGGCTCCTGCTCCTC & CAGGGCCGCCAGCTGGAAAC \\
\hline edn1 - qRT-PCR & GCAAACACACGCTGGCAGAAACA & GAAATCCACGCTTGGCTTCTGTG \\
\hline grem2 - qRT-PCR & GCACACACTCCAGCAGGATGAG & GGAGGCGAGCACTTCTTGTTTGC \\
\hline jag $1 b$ - qRT-PCR & GGCACTGGTACCTGCTGAAGAAC & CCTGGGCTCCTCTGTGGAAATG \\
\hline msxe - qRT-PCR & CGCCTGGCGTTTTCGGTGGA & CCGCGGCTTCCGGTTGGTTT \\
\hline$t b \times 22-2$ - qRT-PCR & CACGCGACAAGTGGATCATA & CACTAACCCTGTGCGTCAAA \\
\hline Wnt3a - qRT-PCR & GGCATCCAGGAGTGTCAGCATC & CCGAGGCTATAGCATGCACGAAC \\
\hline Wnt5b - qRT-PCR & CAGACAGAGGCGATGGAACTGCAG & CGCTCGACTCACAGCATTCACAAC \\
\hline Wnt9a - qRT-PCR & CTCCGTGCCCGGATAGACATGC & GCACGGTGCATGAGCCCGAAAC \\
\hline$d l x 1 a-$ WISH & CCGCAAGCCCCGGACCATATACTC & CTCCGGTGCGACCGGACGAC \\
\hline$d l x 2 a-$ WISH & CСTCACCAACTCCTGCAGATG & GGCAATGATCAACGTGGCATC \\
\hline edn1 - WISH & CTACTACTGGAATACGGGACTTG & CATTTCATAGAAATATCACTCATTAGCC \\
\hline Frzb - WISH & GAGTGAAGGAGATCAGGATTAGAAATC & GCCACCCATTCCTAACACAGC \\
\hline jag1b - WISH & CTGCATTGCTAATGGACAAGTAAC & CACAGGGCAAATAACAGCCTTGGAGTCC \\
\hline pitx2 - WISH & CGAAGATTCGAACGATGACC & TGGGATGTTGAAAAACGAAA \\
\hline sox9a - WISH & GACGTCAATGAGTTTGACCAATACCTC & GGGCATGCAAATTAAGTAGAACATTAC \\
\hline Wnt3a - WISH & ATGATATATCTTGGATATTTCC & CACATGTGTCTAATCTGGTTTTGG \\
\hline Wnt5b - WISH & GGACAACGTCAACTATGGCTAC & CACGTCTGCTACTTGCACAC \\
\hline
\end{tabular}


Wholemount in Situ Hybridization (WISH)

In situs were carried out according to a previously established protocol [68]. Primer sequences used to generate WISH clones are included in the table below. $d l x 1 a, d l x 2 a$, edn1, frzb, jag $1 b$, pitx 2 , sox $9 a$, Wnt3a, and Wnt5b PCR inserts were amplified from cDNA and cloned into the pCRII TOPO vector to allow for bi-directional in vitro transcription. Wnt9a probes were generated from plasmids used in our prior publication [43]. All WISH experiments compared SB-MM (2.6 ng) control injected embryos and SB-MO (2.6ng) injected embryos. Sense and antisense probes were synthesized using Fisher Optizyme polymerase kits and linearized plasmid templates. All probes were synthesized using UTP-digoxigenin (Roche) except for sox $9 a$, which was synthesized using UTP-fluorescein. Probe visualization was carried out using alkaline phospatase staining with NBT and BCIP. After staining embryos were cleared in 100\% glycerol for 24 hours and photographed using brightfield microscopy. Sense RNA probes were tested in uninjected embryos as a negative control to demonstrate fidelity of antisense probes.

Cell Death Assay

$10 \mathrm{ul}$ of acridine orange at a concentration of $0.5 \mathrm{mg} / \mathrm{ml}$ was added to $1 \mathrm{ml}$ of fish water containing embryos. Live SB-MM (2.6 ng) control injected embryos and SB-MO (2.6 ng) injected embryos were incubated in the acridine orange solution for 30 minutes and then destained in fish water for 10 minutes to remove background prior to photographing. Embryos were then embedded in 3\% methyl cellulose on a glass slide, and acridine orange within apoptotic cells was visualized using fluorescent microscopy and a FITC filter.

\section{Results}

Loss of Wnt9b Activity Results in Multiple Craniofacial Phenotypes, including a Foreshortened Face and Jaw Cartilages with an Open Bite

Our prior report of Wnt9b expression demonstrates Wnt9b is expressed maternally, early zygotically and even later during zebrafish craniofacial development [41]. Of particular note Wnt9b is expressed within the first pharyngeal arch (PA1) ectodermal epithelia during the time when CNCC are patterned by the edn1 pathway from Dorsal-Ventral subdomains into Dorsal-Intermediate-Ventral domains. To evaluate the functional role of Wnt9b during these events, a splice-blocking morpholino (SB-MO) was designed to target the splice acceptor site at the boundary of intron 1 and exon 2 in Wnt9b pre-mRNA. SB-MO was predicted to cause deletion of exon 2, resulting in a frameshift and premature stop codon. As a control to validate SB-MO specificity a splice blocking morpholino with 5-base mismatches was also designed and tested (SB-MM). At 5dpf, SB-MM injected control embryo jaws were indistinguishable from uninjected control embryos. However, SB-MO injected embryos displayed mild, moderate and severe malformations (Figures $\mathbf{1}(\mathbf{A})-(\mathbf{L})$ ) of the jaw in a dose dependent manner (Figure 1(M)). Wnt9b morphant abnormalities included anterior foreshortening of the skull with a flat face, fusion of the palatoquadrate-Meckel's (PQ-MK) jaw joint, ventral displacement and puckering of a reduced Meckel's cartilage with medial-directed turning of the palatoquadrate, inversion of the ceratohyal with small or missing ceratobranchials, and absence of swim bladders. These findings of disrupted jaw morphology are consistent with Wnt9b expression in PA1 ectoderm influencing jaw development. Of note, the posterior chondrocranial skull elements around the anterior end of the notochord and the otic vesicles did not display obvious malformations, while anterior chondrocranial elements (e.g. ethmoid) were definitely affected (Figures 1(A)-(L)). The splanchnocranial cartilages of PA2, (e.g. ceratohyal) of PA2 were more mildly foreshortened than those of PA1 (PQ and MK).

Overall, Wnt9b morphant cartilage elements of all three D-I-V domains of the jaws appear foreshortened, although we were most interested in studying upper jaw cartilages from PA1 since mammals exhibiting oral-facial clefting have dysmorphic maxillae. Jaw joint fusion as observed in Wnt9b morphants resembles edn1 pathway mutant zebrafish and is suggestive of disrupted patterning within intermediate and ventral domains while the Wnt9b morphants also displayed a dorsal phenotype to be described in more detail below [45]. The foreshortened skull observed in Wnt9b morphants is similar to skulls within the hammerhead class of mutant zebrafish, including Wnt5b mutants with flat faces [35]. To quantify the observed jaw phenotypes of Wnt9b morphants we developed a jaw severity scoring system based on landmark analysis of alcian blue stained jaw cartilage components (Supplemental Figure 1). Using this scoring system, we determined jaw severity was dose responsive to Wnt9b knockdown and that $100 \%$ of SB-MM control embryos were scored as having normal jaws. It is important to note that embryos with a moderate score have fusion of one jaw joint, while severe scoring embryos have fusion of both jaw joints. The medial turn of the PQ in moderate and severe embryos results from jaw 

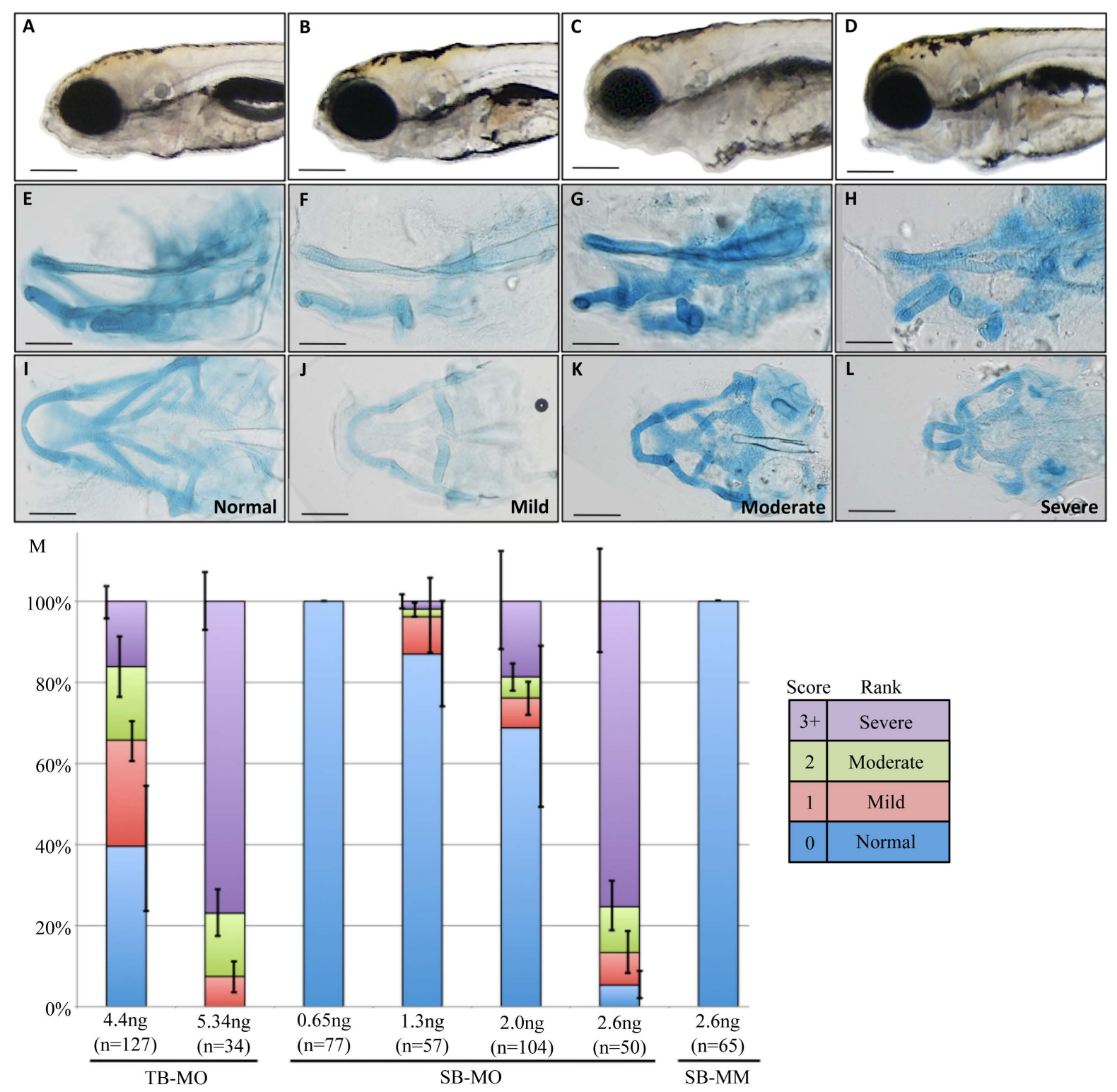

Figure 1. Gross morphological phenotypes of 5dpf zebrafish after Wnt9b knockdown. 5dpf live (A)-(D) and Alcian blue stained (E)-(L) embryos, anterior to left, lateral view (A)-(H) and ventral view (I)-(L). Control embryos, injected with the five base mismatched splice blocking morpholino (SB-MM) are shown within the left most column (A), (E), (I) and are indistinguishable from uninjected controls. Splice blocking morpholino (SB-MO) injected embryos were scored as Mild (B), $(F),(J)$, Moderate $(C),(G),(K)$ or Severe $(D),(H),(L)$ using an objective scoring method (see Supplemental Figure 1. for details) and are shown in subsequent columns. (M) Quantification of the proportion of embryos within each category. Translation blocking (TB-MO), splice blocking (SB-MO) and mismatch control (SB-MM) injected embryos were categorically scored at $5 \mathrm{dpf}$. Injection dose is indicated in ng, and the total number (n) of embryos scored is listed. Note that $5.3 \mathrm{ng}$ TB-MO and $2.6 \mathrm{ng}$ SB-MO produce comparable proportions of affected embryos. Error bars are standard error of means of three biological replicates. Size bars in (A)-(L) are $250 \mathrm{um}$.

fusion and is the cause of MK puckering.

We confirmed through regular RT-PCR from 24hpf cDNA that injection of 2.6ng SB-MO caused deletion of exon 2 (160 bp product from exon 1 to exon 3) while injection of 2.6ng SB-MM did not disrupt Wnt9b transcript processing (414 bp product from exon 1 to exon 3) (Supplemental Figures 2(a)-(b)). We also confirmed through sequencing that the small 160bp band was indeed an aberrant Wnt9b transcript lacking exon 2. We note that in morphants both the normal and disrupted transcript are present, and both at much lower levels than the 
normal band in controls. The apparent reduction in the normal transcript in morphants is likely due to aberrant splicing, while the reduction in the disrupted transcript is likely due to nonsense mediated decay. In support of this we also checked splicing in 28hpf cDNA and noted while both the normal and disrupted transcript were still present, the normal transcript was now obviously more prevalent than the disrupted transcript perhaps due to dilution of the morpholino through cell divisions (data not shown). We found that embryo mortality was also dose responsive to increasing amounts of SB-MO (Supplemental Figure 2(c)). Importantly, 5dpf survival of uninjected controls and 2.6 ng injected SB-MM mismatch controls were similar and both were significantly different from 2.6 ng injected SB-MO embryos.

To further validate our knockdown phenotype's fidelity we designed and tested a translation blocking morpholino (TB-MO) targeting the start codon of the Wnt9b transcript. We similarly evaluated a range of doses and ultimately established that injection of $5.3 \mathrm{ng}$ TB-MO resulted in a similar distribution of jaw phenotypes (mild, moderate, severe) that was comparable to the $2.6 \mathrm{ng}$ dose of the SB-MO. At these respective doses, more than $80 \%$ of embryos injected with $2.6 \mathrm{ng}$ SB-MO or $5.3 \mathrm{ng}$ TB-MO exhibit fusion of one or both jaw joints (Figure 1(M)). We found embryo mortality to TB-MO was also dose responsive. Strikingly, 5.3 ng TB-MO resulted in much greater embryo lethality than $2.6 \mathrm{ng}$ SB-MO, though surviving TB-MO embryos present similar proportions of jaw severity scores (Figure 1(M), Supplemental Figure 2(c)). The increased lethality of the TB-MO relative to the SB-MO at a dose that produces a very similar severity spectrum of jaw phenotypes is consistent with the known differences between translation blocking and splice blocking morpholinos, as TB-MOs block both maternal and zygotic mRNA, while the SB-MOs block only zygotic messages.

As a third control for Wnt9b knockdown specificity, we attempted to rescue Wnt9b morphants by coinjecting Wnt9b morpholinos with synthetic Wnt9b mRNA. We were unable to fully rescue 5dpf jaw defects at multiple tested dose combinations. As a further control to these rescue experiments, we also tested injection of Wnt9b mRNA alone and found overexpression of Wnt9b resulted in ventralization of injected embryos, including loss of dorsal anterior structures like the eye (see below and Discussion).

Wnt9b Morphant Skulls Are Significantly Reduced in A-P Dimension

The flat face phenotype of Wnt9b morphants prompted us to quantitatively assess differences between SB-MO and SB-MM injected embryo skulls. To assess skull length we digitally measured skulls from the landmarks of a line perpendicular to the interhyal joints to the anterior most tip of the Meckel's cartilage from the ventral view. Landmark analysis and measurement revealed Wnt9b morphants have significantly shorter skulls along the anterior to posterior dimension compared to controls (Figures 2(a)-(e)). Categorical ranking was also correlated with skull length with more severe embryos generally having shorter skull lengths, supporting the validity of our categorical rankings. Moreover the measured skull from the interhyal landmark is anterior to PA2, suggesting morphant skull foreshortening is most severe within pharyngeal arch one derivates and the midline ethmoid cartilage.

Wnt9b Morphant Palatoquadrates Are Significantly Shorter in the A-P Dimension

The palatoquadrate (PQ) and specifically the pterygoid process (Ptp) represent the upper lateral zebrafish embryonic jaw element, and as such may embody a patterning process for jaw outgrowth that is homologous between fish and mammals. We therefore wanted to evaluate the extent to which the dorsal PQ and its Ptp might be growing towards the facial midline, as the mammalian embryonic lateral maxillary process does. Therefore in addition to categorically classifying Wnt9b morphant embryos based on severity, we performed fine dissections to remove individual PQ elements for a more detailed analysis. Digital measurements revealed uninjected control or SB-MM injected control embryo PQs were routinely $320.92 \mu \mathrm{m}(+/-19.32 \mu \mathrm{m})$ in length and were not statistically significantly different from each other (Figure 2(f), Figure 2(j)). However, in SB-MO injected embryos we found that even categorically mild embryos displayed PQ lengths of $234.98(+/-25.45 \mu \mathrm{m})$ and were significantly shorter compared to control PQs ( $p$ value $<0.001$ ). We also found that categorical ranking was correlated with PQ length, with more severe ranking generally harboring shorter PQ lengths. Overall, each affected category was significantly different from normal control categories ( $p$ value $<0.001$ ). Mild and moderate PQs were not statistically significantly different ( $p$ value $=0.071$ ), though mild to severe and moderate to severe PQs were significantly different (p value $<0.001$ ).

To determine if the PQ foreshortening in Wnt9b morphants was due to decreased cell number or disrupted migration, we carried out a previously established jaw cartilage morphogenesis assay to determine cell number and cellular orientation [24]. A representative set of mild, moderate and severe palatoquadrate pairs (2, 4 and 4 respectively) from ten different $5 \mathrm{dpf}$ embryos were further dissected out for evaluation of cell numbers and cell 


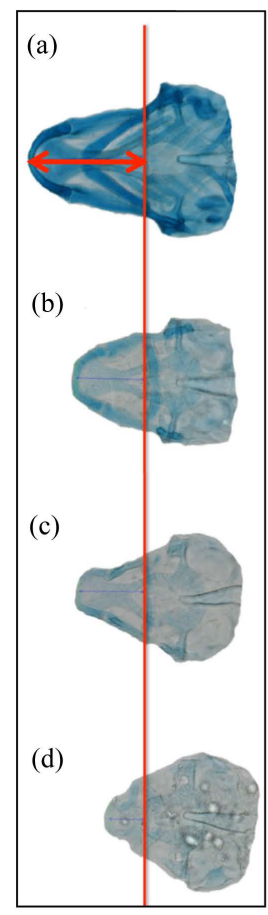

Whole Skull Lengths

(e)

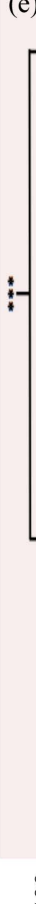

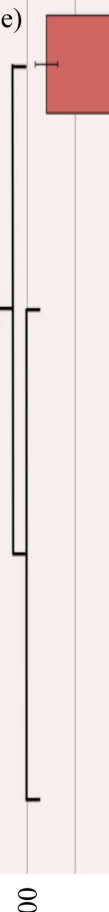

8

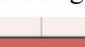

+ lo

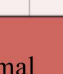

Normal
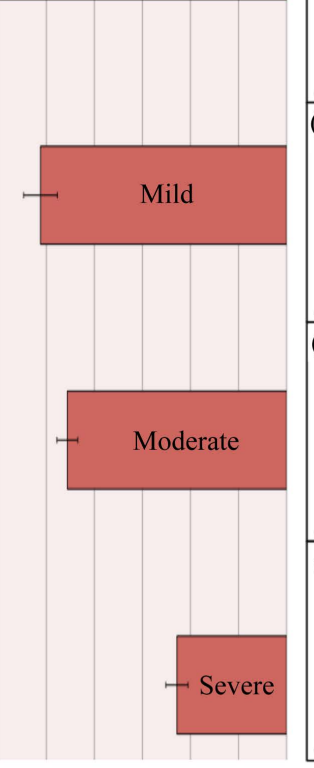

¿ \&

国

皇

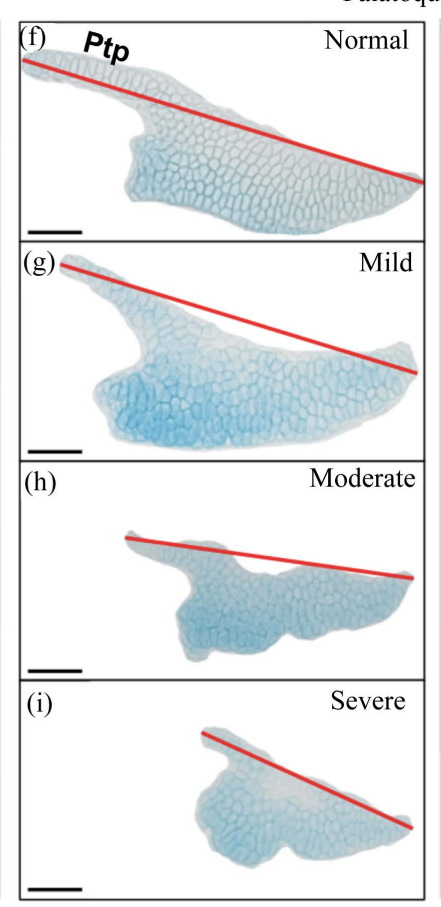

Palatoquadrate Lengths

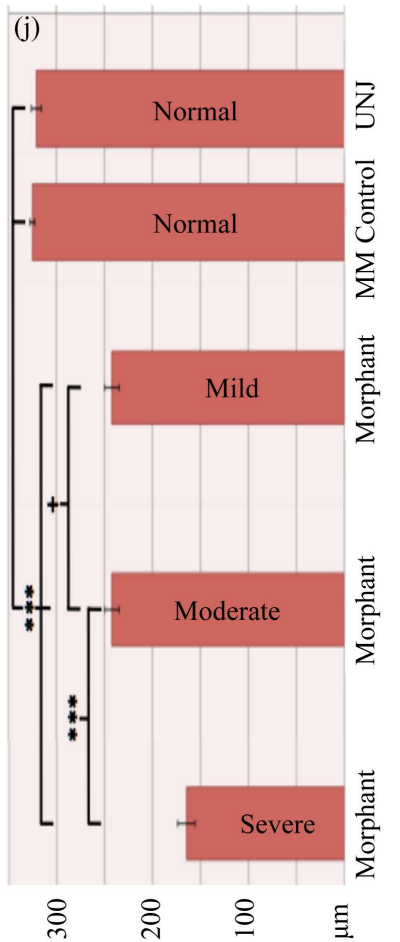

Figure 2. Dissected morphology of Wnt9b morphants reveals significant skull and palatoquadrate (PQ) foreshortening along the anterior-posterior (A-P) axis. Alcian blue whole-skulls (a)-(d) or dissected PQs (f)-(i) were flat mounted and digitally measured. Normal (A), (F) MM control and mild (b), (g), moderate (c)-(h) and severe (d), (i) skeletal elements from SB-MO injected embryos. (a)-(d) Red line aligns these skulls along the common interhyal joint, with digital measurements performed perpendicular to this line to the anterior-most tip of the Meckel's cartilage (red arrows). Note that the anterior tip of the notochords are also aligned. (e) Bar chart showing mean skull lengths per category. All morphant skulls grouped together were significantly shorter than controls. (f) (i) Red line represents the digital measurement of the PQ along the A-P axis. Size bars in (f)-(i) are $40 \mu \mathrm{m}$. (j) Bar chart showing mean PQ lengths per category, and morphant PQs are significantly shorter than controls. Note that uninjected control and MM control PQs are not significantly different. $+\mathrm{p}=0.071$, *** $\mathrm{p}<0.001$. 1-way ANOVA analysis, Tukey’s HSD test of means. Ptp = pterygoid process.

stacking. Most of the PQ cells are polygonal and contact five or six adjacent cells. A side is scored as a "stacked" cell side only if it was noticeably longer than the other sides and if that elongated side was in contact with just one other adjacent cell. Cell numbers and stacked cell sides within each palatoquadrate were counted from digitized photographs (Supplemental Figures 3(a)-(b)). The averaged data from each pair of palatoquadrates were then summed for all morphants and compared to similar data from uninjected control (WT) embryo palatoquadrates. Average PQ cell counts for WT embryos were 235 cells/PQ while from morphants it was 169 cells/PQ. Thus the morphants exhibited a reduction to $71.9 \%$ of WT values, indicating morphants do have a moderate reduction in PQ cells. The WT embryos were found to have 56 stacked sides/PQ while the morphants had only 16 stacked sides/PQ, representing a reduction to $28.5 \%$ in the morphants, suggesting less cell stacking within each morphant PQ. The lack of cell stacking is most evident in the severely scored PQs, as shown in Figure 2(i), which definitely lacks the extended pterygoid process (Ptp). The Ptp of normal PQs exhibit a high degree of cell stacking (Figure 2(f)). Disrupted cell stacking in Wnt9b morphants is suggestive of disruption of convergent extension processes [69].

Wnt9b Knockdown Disrupts D-V to D-I-V CNCCs Patterning Genes within the First Pharyngeal Arch

To assess potential genetic interactions with Wnt9b, we performed qRT-PCR in SB-MM control and Wnt9b SB-MO injected embryos aged to $24 \mathrm{hpf}$ or $28 \mathrm{hpf}$. At the onset of D-I-V jaw patterning at 24hpf, Wnt9b morphants exhibited down regulated expression of D-I-V spanning (dlx2a) and I-V spanning (edn1, tbx22, msxe) as well as down regulated expression of Wnt pathway related genes (Wnt3a, Wnt5b, Wnt9a, and axin2) (Figure 3(a)) [20] [44]. After the onset of D-I-V patterning at 28hpf we found similar patterns for I-V spanning genes as 
(a)
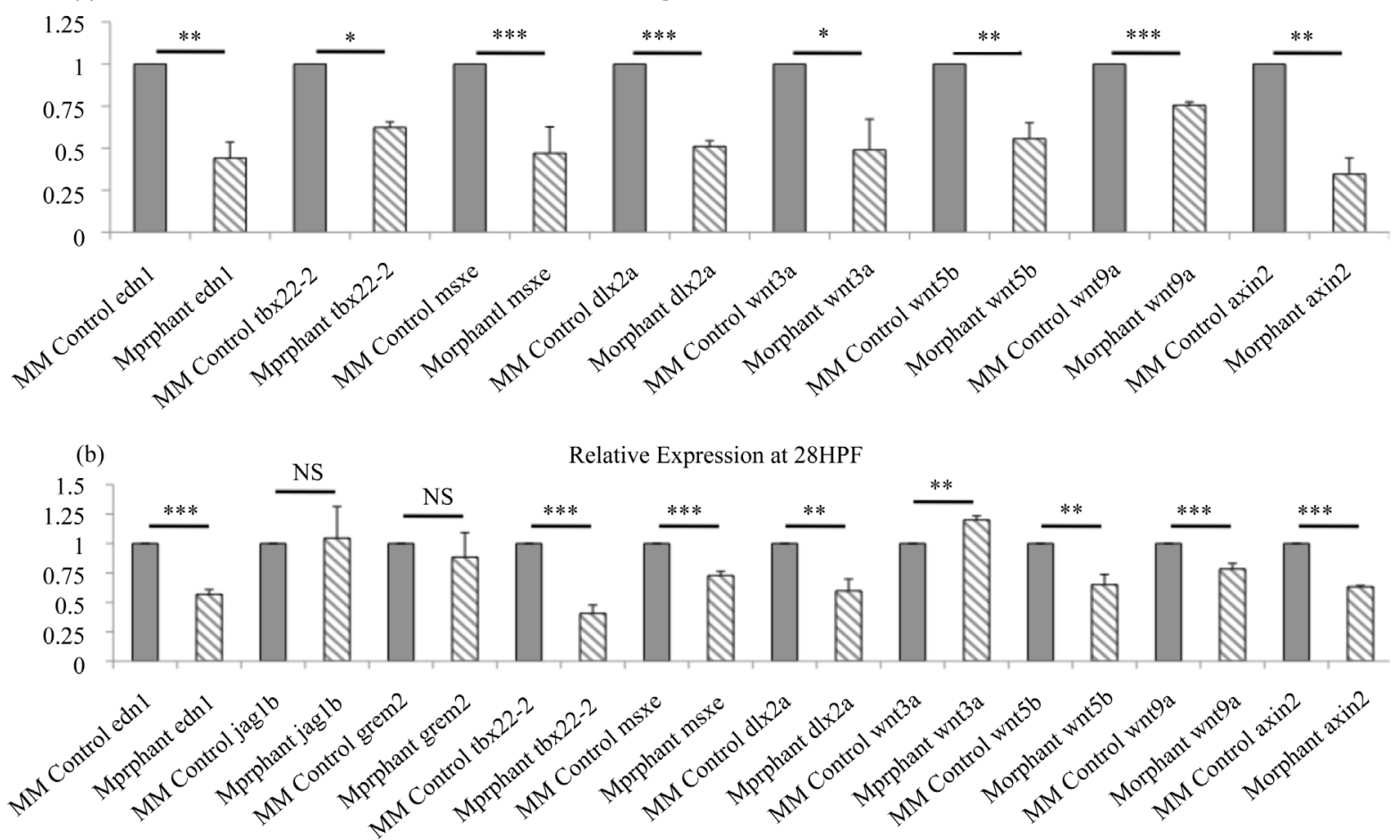

Figure 3. Wnt9b knockdown alters global expression of known D-I-V patterning genes as well as other Wnt pathway genes. Embryos were injected with $2.6 \mathrm{ng}$ of SB-MM or SB-MO and qRT-PCR was performed on cDNA from 24hpf (a) or 28hpf (b) samples. Error bars are the standard error of fold changes of three biological replicates. Significance was calculated using a T-test, NS = Not Significant, ${ }^{*} \mathrm{p}<0.05,{ }^{* *} \mathrm{p}<0.01,{ }^{* * *} \mathrm{p}<0.001$.

well as for $d l x 2 a$, though we did not note changes in dorsal spanning genes grem2 or jag1b (Figure 3(b)) [51]. These data are consistent with Wnt9b being at least partially upstream of the whole edn1 pathway that has been shown to pattern PA1 CNCCs from an early D-V to eventual D-I-V domains. At 28hpf we found a similar pattern for Wnt pathway genes compared to 24hpf with the exception that Wnt3a expression was increased at $28 \mathrm{hpf}$. These data suggest Wnt9b is influencing Wnt pathway signaling at 24hpf and 28hpf.

As qRT-PCR can only measure fold expression changes globally, we next decided to perform whole-mount in situ hybridization (WISH) on SB-MM control and SB-MO injected embryos to determine more subtle spatial and temporal expression changes. Confirming our edn1 qRT-PCR data, in situ hybridization revealed reduced edn1 expression in PA1 in 63\% of Wnt9b morphants (Figures 4(a)-(d)). Though we did not find jag1b expression changed at 28hpf, by 48hpf jag1b was dramatically expanded ventrally similar to findings in edn1 deficient embryos (Figures 4(e)-(h)) [52]. These results further validate that edn1 signaling is genuinely disrupted in Wnt9b morphants and suggests Wnt9b acts upstream of edn1 to promote ventral and intermediate identities.

We next assessed D-I-V spanning $d l x 2 a$ expression in Wnt9b morphants and found $d l x 2 a$ was strongly reduced in PA1 and PA3-7 in 60\% of Wnt9b morphants (Figures 5(a)-(d)). dlx2a was most strongly reduced in the dorsal domains of the PAs, with weak ventral expression still visible. $d l x 2 a$ expression in PA2 was not strongly down regulated, though $d l x 2 a$ within PA2 is known to be downstream of tfap2a [70] [71]. Moreover, $d l x 2 a$ expression in the brain was unaffected, suggesting $d l x 2 a$ is mostly dependent on Wnt9b signaling within PA1 and PA3-7. $d l x 2 a$ and $d l x 1 a$ may potentially be regulated in cis, as they are found adjacent in a digene cluster in vertebrates. We therefore also assessed $d l x 1 a$ expression in Wnt9b morphants. At 24hpf we found $d l x 1 a$ expression in the PAs was very weak relative to $d l x 2 a$ expression, even in control embryos, and were unable to discern a difference between test and control specimens (data not shown). We therefore were unable to definitively determine if PA $d l x 1 a$ expression was dependent on Wnt9b signaling.

We next wanted to determine if disruption of patterning gene expression in Wnt9b morphant PAs was due to increased cell death. We assayed cell death in live Wnt9b morphants and controls using acridine orange staining. This assay did identify increases in cell death within dorsal neural tissues including the rhombic lips and the 


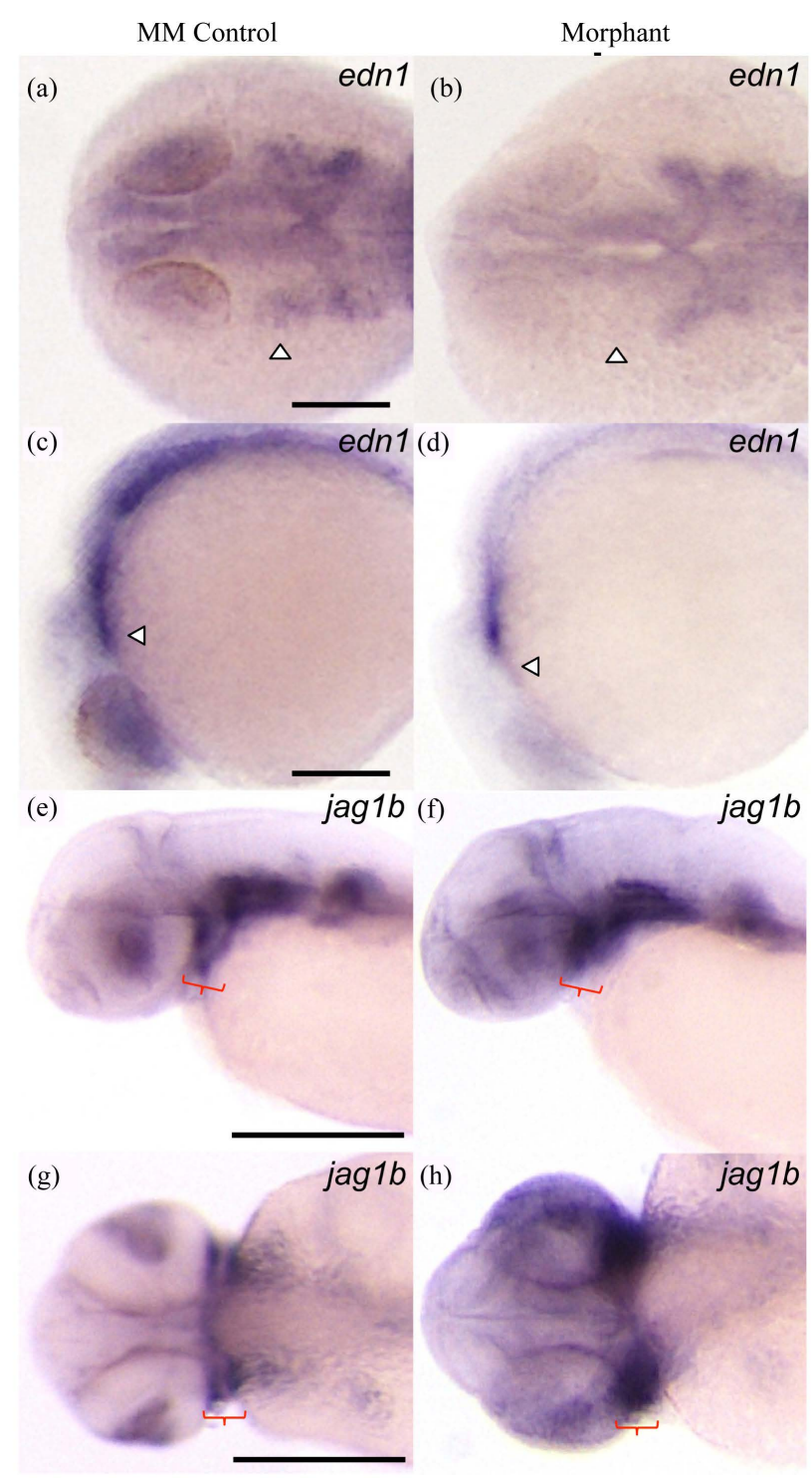

Figure 4. Wnt9b morphants exhibit moderate reduction of edn1 expression and the expected expansion in jag1b expression in the pharyngeal arches. (a)-(d) Anterior to right, top row = dorsal view, bottom row = lateral view. MM control $(\mathrm{n}=8)(\mathrm{a})$, (c) and morphant $(\mathrm{n}=8)(\mathrm{b}, \mathrm{d})$ embryos were aged to 24hpf and stained with edn 1 antisense riboprobe. $63 \%$ of morphant embryos exhibited reduced edn 1 expression in the pharyngeal arches compared to controls. (e)-(f) Top row = lateral view, bottom row $=$ anterior view. jag1 $b$ expression in 48hpf aged MM control $(n=14)(e),(g)$ and morphant $(n=10)(f)-(h)$ embryos. $100 \%$ of morphant embryos exhibited expanded jag $1 b$ expression in the pharyngeal arches compared to controls. White arrowheads indicate PA1. Red brackets indicate PA1. Size bars $=100 \mu \mathrm{m}$.

brain (Supplemental Figures 4(a)-(b)). There were, however, very minimal differences in cell death within the PAs of controls and Wnt9b morphants.

Through qRT-PCR we noted changes in Wnt pathway genes, so we further assessed expression of Wnt3a and Wnt5b (Figures 6(a)-(d)). 71\% of Wnt9b morphants demonstrated increased expression of Wnt3a dorsally both 


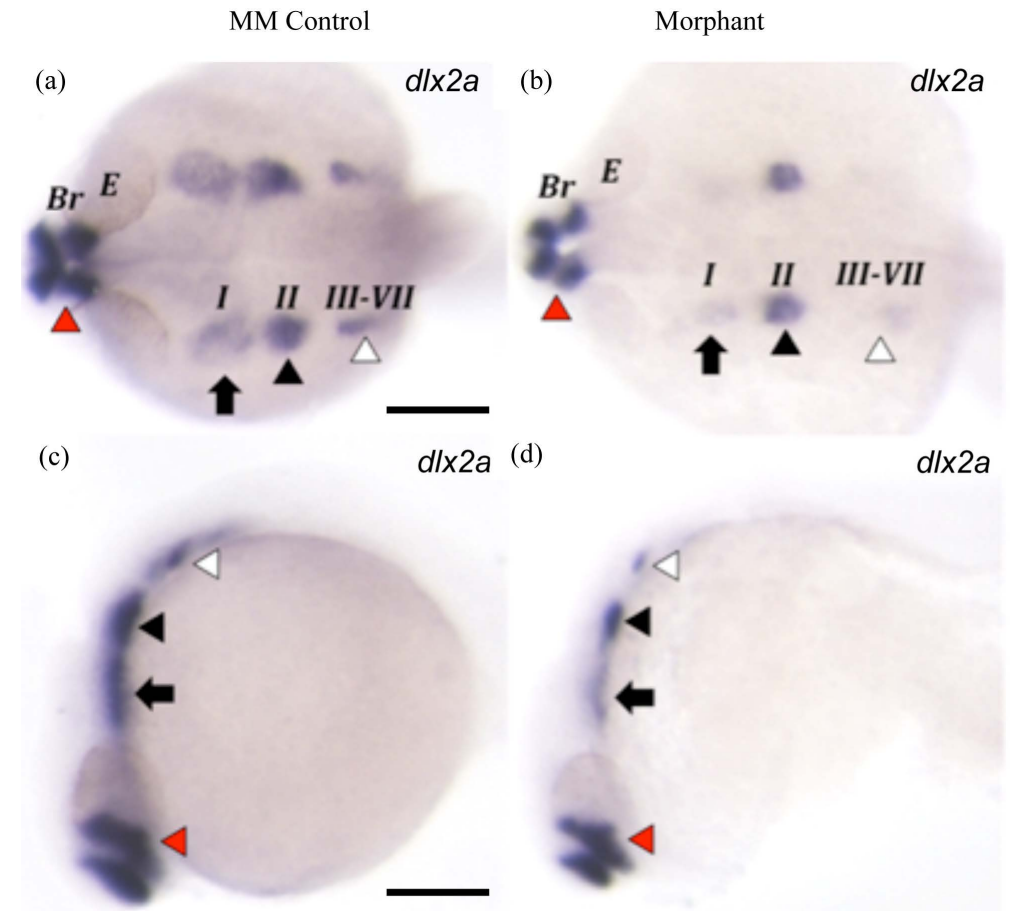

Figure 5. Wnt9b morphants exhibit severely reduced $d l x 2 a$ expression in pharyngeal arches 1 and 3-7. (a)-(d) Anterior to right, top row = dorsal view, bottom row = lateral view. $M M$ control $(n=17)(a),(c)$ and morphant $(n=20)(b),(d)$ embryos were aged to $24 \mathrm{hpf}$ and stained with $d l \times 2 a$ antisense riboprobe. $60 \%$ of morphant embryos exhibited reduced $d l \times 2 a$ expression in the pharyngeal arches compared to controls. Red arrowheads indicate forebrain. Black arrows indicate PA1, black arrowheads indicate PA2, white arrowheads indicate PA3-7. Note that $d l x 2 a$ expression is mostly reduced in PA1 and PA3-7. $B r=$ brain, $E=$ eye. Size bars $=100 \mu \mathrm{m}$.

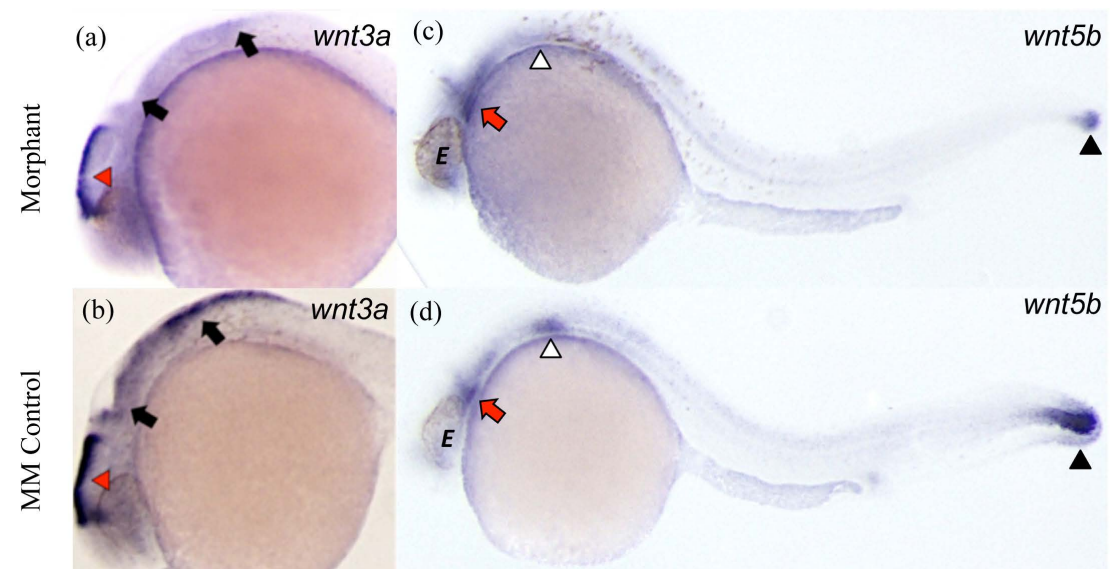

Figure 6. Wnt9b morphants exhibit disrupted Wnt3a and Wnt5b expression domains. Anterior to left. Left column is Wnt3a expression, right column is Wnt5b expression. (a) MM control ( $\mathrm{n}=14)$ and (b) morphant embryos ( $\mathrm{n}=14$ ) were aged to $28 \mathrm{hpf}$ and stained with Wnt3a riboprobe. $71 \%$ of morphant embryos exhibited expanded Wnt3a expression in the brain compared to controls. (c) MM control $(n=10)$ and $(d)$ morphant embryos $(n=9)$ were aged to $28 \mathrm{hpf}$ and stained with Wnt5b riboprobe. $100 \%$ of morphant embryos exhibited expanded Wnt5b expression in the tail with reduced Wnt5b expression in the pharyngeal arches compared to controls. (a), (b) Red arrowheads indicate brain expression, black arrows indicate rhombic lip expression. (c), (d) Red arrows indicate the PAs, white arrowheads indicate the fin buds, black arrowheads indicate the tail. 
within the brain and rhombic lips, two sites where we noted increased apoptosis (Supplemental Figure 4). This increased expression of Wnt3a shown by WISH also validated the Wnt3a result observed via RT-PCR at 28hpf. These results suggest the Wnt9b morphant jaw phenotype is not predominantly caused by increased cell death within the PAs and reveal an unexpected effect of Wnt9b signaling on dorsal neural tissues. In addition Wnt5b was upregulated in the fin buds and dorsal axial tail but down regulated within the ventral pharyngeal arches in 100\% of Wnt9b morphants (Figure 6(c), Figure 6(d)). These results are consistent with Wnt9b inhibiting dorsal body axis genes like Wnt3a and Wnt5b while stimulating genes within the ventral half of the head, such as edn1.

Wnt9b Deficiency Disrupts Transition to the Secondary Mouth and its Jaws and Face

Wnt9b morphants share similar skull abnormalities to the hammerhead class of mutants [35]. Among these mutants, the zebrafish pipetail mutant (Wnt5b) was particularly interesting to us due to known roles for Wnt5a in murine midfacial outgrowth and palatal development and Wnt5a association to human OFC [12] [34]. We assessed Wnt5b expression after opening of the primary mouth, during the initial outgrowth phases of the secondary mouth (Figures 7(a)-(f)). Close inspection of pharyngeal arch 1 derivatives revealed that in controls, Wnt5b expression was strongest at the growing tip of the pterygoid process (Ptp) and was conspicuously absent from the posterior body of the PQ. The Ptp was seen as a growing rod of cells, perhaps intercalating and stacking towards the midline ethmoid reminiscent of convergent extension of chondrocytes during long bone outgrowth [69]. In 85\% of Wnt9b morphants, however, Wnt5b expressing cells of the presumptive Ptp were clumped adjacent to the posterior body of the PQ, unmistakably failing to extend toward the midline ethmoid.

We next assessed whether palatoquadrate chondrocyte differentiation was affected in Wnt9b morphants by assaying sox $9 a$ through WISH [72]. We found that sox9a was downregulated within the dorsal ethmoid as well as first pharyngeal prechondrocyte condensations in 73\% of Wnt9b morphants (Figure 7(g), Figure 7(h)). These findings are also consistent with a loss of $d l x 2 a$ expression, which is required for sox $9 a$ expression in the PAs [54].

Due to demonstrated roles for Wnt9a in ethmoid growth and sox $9 a$ expression, we next sought to determine whether Wnt9a expression was changed in Wnt9b morphants [73]. Despite modest reductions in Wnt9a expression observed through qRT-PCR, we were unable to demonstrate any obvious changes in Wnt9a spatial expression domains after Wnt9b knockdown at either 28hpf or 48hpf via WISH (data not shown). We therefore hypothesize that Wnt9a may be acting predominantly through a different and parallel pathway to Wnt9b during jaw formation.

Wnt9b Signaling Is Required for Proper Transition from the Primary to Secondary Mouth

A recent study demonstrated that pitx2 deficiency resulted in craniofacial anomalies in zebrafish [74]. Moreover it has been shown that pitx2 is a target for C-Wnt and that pitx2 is a marker for primary mouth formation [57]. We therefore evaluated its expression in Wnt9b morphants. We found that in $82 \%$ of Wnt9b morphants, pitx2 expression was dramatically increased within the inner aspects of the initial phase of secondary mouth outgrowth (Figures 8(a)-(d)). We take these results to suggest that Wnt9b normally represses pitx2 and its role in the primary mouth, perhaps facilitating the transition to the secondary mouth outgrowth phase by helping terminate primary mouth gene networks.

Wnt9b May Affect Initiation of the Primary Mouth Just after Gastrulation

Wnt antagonists from the dorsal anterior mesoderm, including preplacodal plate mesoderm, help to maintain the preplacodal ectoderm region that ultimately leads to the oral placode and primary mouth [64]. We therefore checked the midline Wnt antagonist frzb found within the preplacodal plate, or the zebrafish polster. In 93\% of Wnt9b morphants we found that frzb expression was dramatically expanded (Figures 9(a)-(d)). These results are consistent with Wnt9b playing an early role in modulating the Wnt inhibitor frzb to control induction or maintenance of the primary mouth. Further, this is a third example of Wnt9b inhibiting dorsal Wnt pathway expression domains, beyond those described above (Wnt3a and Wnt5b).

Based on the interaction of Wnt9b with frzb expression, we surmised that overexpression of Wnt9b would reduce dorsal anterior structures. We found that overexpressing synthetic Wnt9b reduced dorsal anterior derived structures and loss of anterior extension in a dose dependent manner (Supplemental Figure 5).

\section{Discussion}

Wnt9b Patterns the CNCC into D-I-V Jaw Domains:

Wnt9b contributes to CNCC patterning within the 1st Pharyngeal Arch 

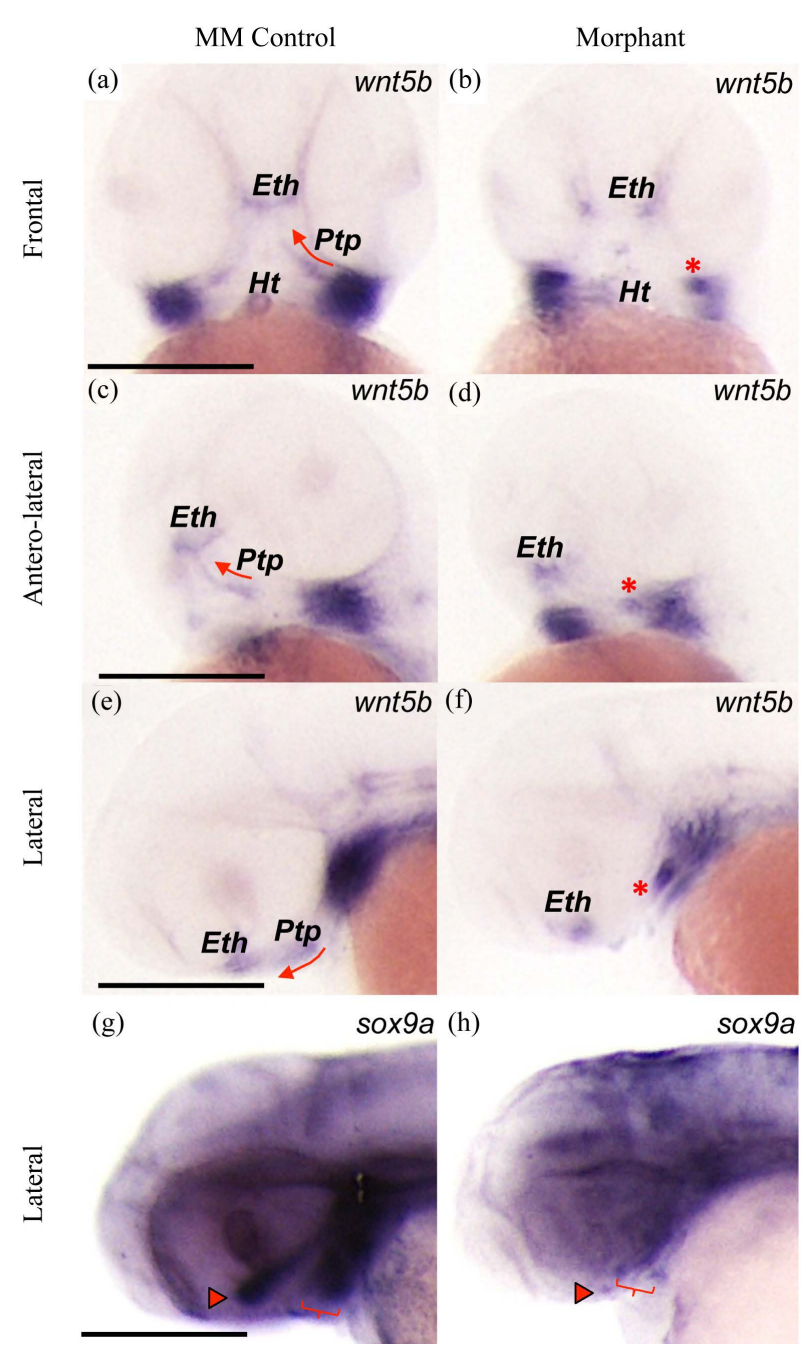

\begin{abstract}
Figure 7. Wnt9b morphants display disrupted Wnt5b and sox9a expression domains in the developing upper jaw. MM control ( $n=11)$ (left column) and morphant $(n=13)$ (right column) embryos were aged to 48hpf and stained with Wnt5b riboprobe (a)-(f) or sox $9 a$ riboprobe (g), (h). $85 \%$ of morphant embryos exhibited disrupted Wnt5b expression in the pharyngeal arches compared to controls. $73 \%$ of morphant embryos exhibited reduced sox $9 a$ expression in the pharyngeal arches compared to controls. Frontal views (a), (b), antero-lateral views (c), (d), and lateral views (e)-(h) with anterior to the right. Red arrows trace the linear outgrowth of the pterygoid process. Red asterisks indicate clumped pterygoid process cells. Red arrowheads indicate the ethmoid. Red brackets indicate the first pharyngeal arch precartilage condensations. $E=$ eye, $E t h=$ ethmoid, $H t=$ heart tube, $P t p=$ pterygoid process. Size bars $=100 \mu \mathrm{m}$.
\end{abstract}

We are the first to demonstrate that Wnt9b contributes to the edn 1 signaling pathway within the pharyngeal arches. Wnt9b expression within pharyngeal arch ectodermal epithelia coincides spatially and temporally with edn1, during the crucial window when edn1 is acting to pattern the D-I-V subdomains within PA 1 and 2 . Adding to previous studies of edn1 signaling, we demonstrated that Wnt9b morphants had reduced expression of ventral and intermediate patterning genes as well as increased dorsal patterning gene expression of $j a g 1 \mathrm{~b}$, consistent with reports using edn1 mutants [52]. edn1 was only moderately reduced in Wnt9b morphants, suggesting that either other factors contribute to edn1 expression or the Wnt9b morpholino is only partially effective at this dose, as suggested by the reduced but still present wild type Wnt9b transcript (Supplemental Figure 2). Alternatively, edn1 expression was previously demonstrated to be downstream of $b m p 4$, so perhaps Bmp and Wnt signaling are combinatorial to promoting edn1 expression within the PAs [75]. Consistent with partial 
MM Control

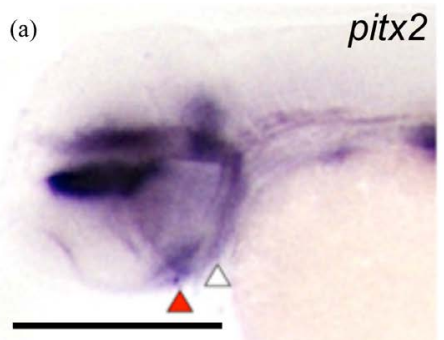

(c)

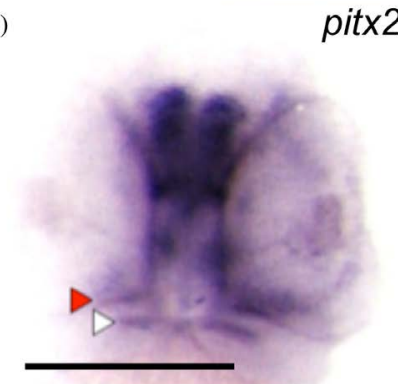

Morphant

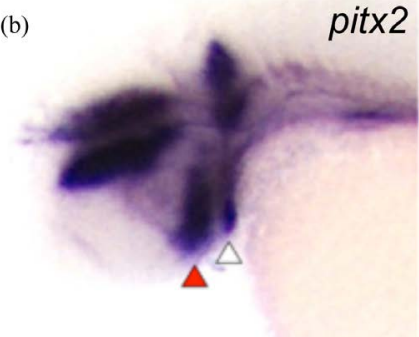

(d)

pitx2

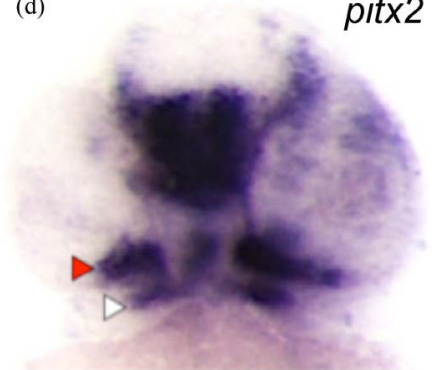

Figure 8. Wnt9b morphants exhibit expanded pitx2 expression domains in the developing mouth. Top row = anterior to left, bottom row = anterior to front. MM control $(\mathrm{n}=8)(\mathrm{a})$, (c) and morphant $(\mathrm{n}=11)(\mathrm{b})$, (d) embryos were aged to $48 \mathrm{hpf}$ and stained with pitx2 riboprobe. $82 \%$ of morphant embryos exhibit expanded pitx2 expression within medial oral regions compared to controls. Red arrowheads indicate upper jaw, white arrowheads indicate lower jaw. Size bars $=100 \mu \mathrm{m}$.

MM Control

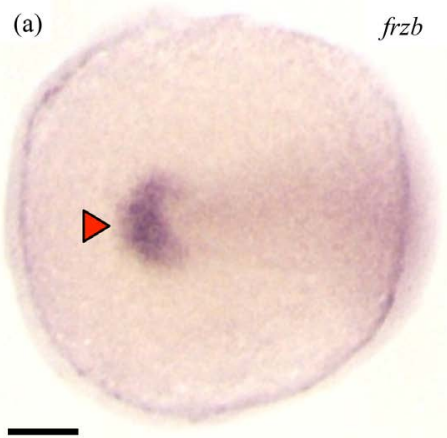

(c)
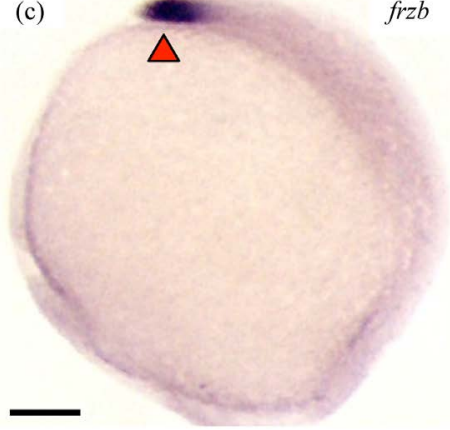

Morphant

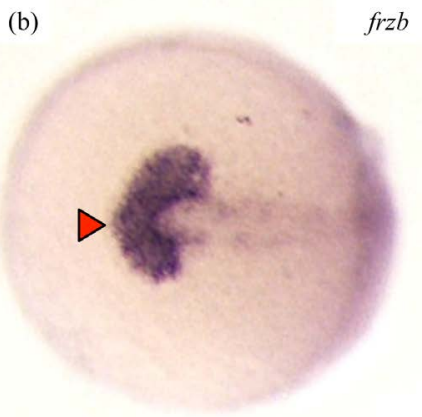

(d)

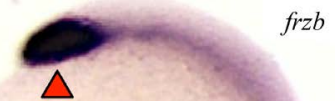

frzb

Figure 9. Wnt9b morphants exhibit expanded frzb expression in the polster. Top row = dorsal view, bottom row = lateral view, anterior to left. MM control $(n=6)(a),(c)$ and morphant $(n=15)(b)$, (d) embryos were aged to $13 \mathrm{hpf}$ and stained with frzb riboprobe. $93 \%$ of morphant embryos exhibit expanded frzb expression compared to controls. Red arrowheads indicate the polster. Note that morphants have upregulated and expanded expression of frzb. Size bars $=100 \mu \mathrm{m}$. 
reduction of edn1, Wnt9b morphant jaws exhibit comparably mild defects of the jaw joint and Meckel's cartilage compared to those observed in edn1 mutants. While the present findings in zebrafish are interesting, it is unclear whether the same correlations can be drawn in mammals. For instance, consider the similarities between edn1 mutant zebrafish and Edn1 mutant mice [46]. Both mutants show presumptive homeotic transformations of the functional equivalents of the lower jaw into upper jaw identities. Even though the functional equivalents of the lower and upper jaw may be anatomically unrelated in these two species (endochondral versus dermal bone origin), loss of Edn1 signaling nonetheless affects these adult jaws in highly similar fashions. Therefore the homology of the jaws lies not in the derived anatomical structures (i.e. the palatoquadrate contributes strongly to adult jaws in fish but mostly to ear ossicles and the alisphenoid in mammals) but instead in the evolutionarily conserved gene-regulatory networks that govern jaw, face and secondary mouth patterning. Wnt9b deficiency disrupts mammalian jaw formation, and if Wnt9b contributes to a conserved facial gene-regulatory network (like Edn1 does) then it would be expected that loss of Wnt9b in zebrafish would result in a functionally analogous phenotype.

\section{Reductions of dlx2a expression are predictive of jaw cartilage defects}

Previous studies of genes modulating $d l x 2 a$ show that PA regions with compromised $d l x 2 a$ expression show more severe disruptions in jaw cartilage morphology. For example in zebrafish tfap2a mutants dlx2a is strikingly absent within PA2 but not within PA1 [70] [71]. Importantly jaw cartilage defects within tfap2a mutants are most severe within PA2, consistent with PA2 showing the most disrupted $d l x 2 a$ expression.

Loss of either Dlx 1 or Dlx 2 or both in mice leads predominantly to proximal (dorsal) defects in the derivatives of PA1 and PA2, consistent with the Dlx code [55] [56]. This includes proximal dermatocranial structures (maxillary, palatine, pterygoid) as well as proximal splanchnocranial structures (the proximocaudal incus and the rostral ala temporalis, AT). The latter is the embryonic cartilage that ultimately contributes to the endochondral aspect of the alisphenoid, the greater wing of the sphenoid bone in mammals. The embryonic AT and adult alisphenoid are homologous to the middle portion of the palatoquadrate, the endochondral jaw maxillary cartilage of the fish [21].

Dlx2 mutant mice and Wnt9b mutant mice share some similarities with regards to these proximal endochondral and dermal derivatives, in addition to both having cleft secondary palate. Although not described in their report, Wnt9b mutant mice also show an apparently reduced alisphenoid and palatine, besides exhibiting clefting of the more distal maxilla and premaxilla [39]. Wnt5a mutant mice also have cleft secondary palate, though these mutant reports did not examine if Wnt9b and Wnt5a mice have reductions in the endochondral ala temporalis.

In mammals, both the V2 (maxillary) and V3 (mandibular) branches of the trigeminal nerve course through the alisphenoid via the foramen rotundum and ovale, respectively. This places the alispenoid at the caudal base of the jaws in mammals, with only dermal jaw bones found more anteriorly. Note that the Dlx code patterns Dorsal to Ventral structural differences within the pharyngeal arches but does not disrupt nor pattern the structural differences along the Anterior to Posterior axis.

In Wnt9b morphants, $d l x 2 a$ expression within PA1 was absent in the dorsal domain and weak in the ventral domain. Conversely, in edn1 mutant zebrafish, $d l x 2 a$ expression is absent in the ventral domains and unaffected in the dorsal domains [45]. The severity of affected jaw cartilages in Wnt9b morphants and edn1 mutants demonstrates that lack of $d l x 2 a$ in a specific domain greatly affects jaw cartilage morphogenesis within that respective domain. Wnt $9 \mathrm{~b}$ and $e d n 1$ are both secreted signals from the PA1 ectodermal epithelia that overlie $d l x 2 a$ expressing CNCC. Given the current understanding of $e d n 1$ and $d l x 2 a$ signaling, it was suggested $d l x 2 a$ in the ventral domains is promoted by edn 1 signaling from the ectoderm. The dorsal $d l x 2 a$ domain, however, was unaffected in edn1 mutants. The Wnt9b morphant phenotype data presented above includes reductions in both dorsal (PQ) as well as ventral (MK) cartilage elements. This is consistent with reduced signaling through $d l x 2 a$, responsible for the reduction in dorsal (proximal) elements, as well as reduced signaling through the edn1 pathway, responsible for disruption to the I and V domains of PA1.

It is interesting that Wnt9b morphants display jaw cartilage defects mostly within derivatives of PA1 and PA3-7, and components of the hyoid arch were more mildly affected. Further, WISH of edn1 and $d l x 2 a$ in the Wnt9b morphants revealed the most severe reductions in PA1 and PA3-7. Yet PA2 reduction of the ceratohyal cartilage was also observed. We postulate that this PA2 reduction is downstream of a reduced regional edn1 signal subsequent to Wnt9b knockdown, as edn1 signals to both PA 1 and 2.

Wnt9b Promotes Anterior Outgrowth of the Jaws 
Severely Stunted Outgrowth in Wnt9b Deficient Upper Jaws

Wnt9b morphant skulls and notably the upper jaw display a significant anterior-directed growth deficiency. Not only are whole morphant skulls shorter, but the PQ is greatly reduced along the anterior to posterior axis. Shorter length and size may be attributed to multiple underlying causes ranging from reduced proliferation to increased apoptosis to stunted migration, all of which may be downstream of the earlier patterning events.

The lost anterior outgrowth observed in Wnt9b morphants is at least partially due to fewer cells contributing to the upper jaw. Quantitative assessment of morphant PQs showed approximately 1/3 fewer cells with a $1 / 2$ overall decrease in length. This may result from less proliferation or increased apoptosis. However, the cell death assay revealed no change in cell death within morphant pharyngeal arches compared to controls. This argues against Wnt9b morphants having fewer cells due to increased cell death. Wnt9b morphants show reductions in msxe expression. Combinatorial knockdown of $m s x b / m s x c / m s x e$ results in ablations of most PA1 derived jaw cartilage elements [76]. msxe promotes cell proliferation within the pharyngeal arches, and reduced proliferation within PA1 could explain why Wnt9b morphant PQs have fewer cells. Consistent with conserved patterning influences, Wnt9b mutant mice also show reduced PA1 expression of Msx 1 , the mammalian ortholog of msxe [77]. Further, Msx1 mutations in humans result in oral facial clefting, with deficient directed growth of the maxilla [8], thus supporting conservation of gene regulatory networks downstream of Wnt9b for facial outgrowth.

An additional factor that perhaps contributes to PQ length is convergent extension [69]. It was found that Wnt9b morphants had irregular cellular orientation within the PQ and its Ptp (Supplemental Figure 3). Especially relevant and additive to this finding was that Wnt5b expression was abnormal within the PQ (Figure 7). One reason for disrupted cartilage cell orientation in Wnt9b morphants may be that the cells are thus unable to undergo appropriate morphogenetic changes and therefore cannot appropriately interpret oriented migrational cues into directed outgrowth.

One other notable morphological discrepancy between Wnt9b morphants and especially edn1 D-I-V pattering mutants is the apparent length of the Ptp relative to the whole PQ length. The Ptp process of the PQ is not foreshortened in edn1 pathway mutants, as opposed to the present results where we demonstrated a definitive foreshortening of this maxillary element. Modulation of the dorsal PA marker jag1b may affect PQ length, though the changes appear confined to the posterior PQ and not the Ptp [52]. Therefore jag1b expression is not likely required for Ptp extension. Wnt9b expression persists dorsally within PA1 through at least 40hpf as it comes to surround the primary mouth and is thus well placed to influence outgrowth and morphogenesis of the Ptp, perhaps via convergent extension processes typically associated with the non-canonical role of Wnt5b [36]. Wnt9b knockdown results in dramatic shortening of the Ptp by 5dpf in fish and this finding is reminiscent of disrupted maxillary outgrowth in Wnt9b deficient mice as well as in human oral-facial clefting patients [37] [78].

Here it is worth noting that Wnt5b mutant zebrafish exhibit a hammerhead skull phenotype with anterior foreshortening of jaw cartilage elements [35]. Also noteworthy is that Wnt5a mutant mice have reduced anterior growth of the midface as well as cleft palate resulting from deficient medial migration within the palatal shelves [34]. Murine Wnt5a exhibits an expression gradient, with stronger expression at the growing anterior tips of the palate and weaker expression in the posterior palate. This finding shares striking similarity to our finding of Wnt5b expression in the zebrafish jaw; Wnt5b expression was stronger in the anterior growing tip of the Ptp and weaker in the posterior body of the PQ. While Wnt5b expression was not absent in Wnt9b morphant Ptps, the morphant Ptp morphology was obviously clumped as a ball of cells and not rod-like as in control Ptps. Taken together these results may suggest Wnt5b expressing Ptp cells have reduced convergent extension in Wnt9b morphants and this causes lack of anterior Ptp morphogenesis toward the midline ethmoid. It would be interesting to determine if Wnt9b knockout mice exhibit similar disruption of Wnt5a or Wnt5b expression in the lateral maxilla.

Primary to Secondary Mouth

The primary mouth is marked by expression of PITX genes, and recent studies in zebrafish show that pitx2 deficiency disrupts jaw cartilage morphology [74]. Wnt9b morphants show marked upregulation and expansion of pitx 2 within the oral regions. Previous studies of the primary mouth show that inhibitors of canonical Wnt signaling are required prior to primary mouth formation [64]. In Xenopus, these inhibitors include frzb. Our results above showed that Wnt9b modulates early frzb expression, at least consistent with a potential downstream effect upon the primary mouth. This area needs much more work before the mechanism for this influence can be understood. 
The reports on primary mouth formation in Xenopus also demonstrated that overexpression of canonical Wnts decreased the size of the primary mouth [64]. Our results above demonstrate that Wnt9b normally inhibits pitx2 expression after the primary mouth has opened. If Wnt9b signals through a canonical Wnt pathway to the primary mouth to turn off this pitx2 expression, the present results may provide a mechanism to explain the influence of canonical Wnt signals upon primary mouth markers like pitx2. Perhaps this Wnt9b inhibition of pitx2 facilitates the transition from the primary to the secondary mouth.

\section{Conclusions}

When considering the new implications this study provides for human OFC disorders, it is important to discuss differences and similarities in facial development of different vertebrate taxa. Loss of Wnt9b in mice is very closely phenocopies human OFC. Humans and mice are, however, both mammals and therefore share much more structural homology in the jaws compared to zebrafish. Zebrafish jaw elements cannot be directly compared to mammals due to several derived characteristics gained long after separation from the zebrafish lineage. These derived characteristics suggest the anatomical jaw elements in zebrafish and mice are structurally unrelated. The upper and lower jaw equivalents of mammals and zebrafish, despite lacking anatomical homology, may nonetheless be affected similarly by Wnt9b deficiency, perhaps through developmental pathways that include a Wnt9 to Wnt5 activation signal. We therefore argue that the homology lies not strictly in the anatomical structures but within the gene-regulatory network that patterns jaw precursors prior to the later derived developmental events. The evidence provided in this study strongly supports this notion.

The results of our expression studies suggest Wnt9b signaling generally promotes ventral (anterior-ventral facial ectodermal expression and facial patterning) while antagonizing dorsal patterning (Wnt3a, Wnt5b, and frzb). Wnt9b morphants demonstrate increased dorsal expression of Wnt3a within the dorsal hindbrain, Wnt5b within the axial tail, and increased frzb within the dorsal prechordal plate. Consistent with these observations, when Wnt9b was overexpressed by itself, we observed ventralization of embryos. This is very similar to what had been reported when the Drosophila Wnt9 ortholog, DWnt4, was over expressed in Xenopus embryos (Supplemental Figure 5) [79]. Importantly these ventralized phenotypes were never observed upon injection of morpholino alone, and the proportion of ventralized embryos was dose responsive to Wnt9b mRNA. These data suggest Wnt9b mostly promotes outgrowth to the anterior-ventral half of the head.

Figure 10 summarizes a newly proposed model of Wnt9b signaling during zebrafish facial development.

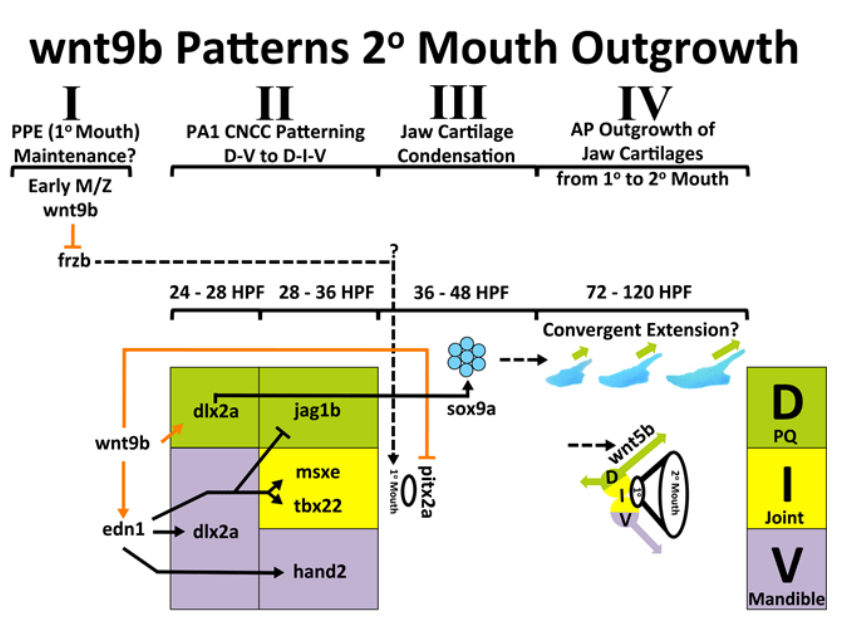

Figure 10. Wnt9b patterns $2^{\circ}$ mouth outgrowth. (I) Maternal and early zygotic Wnt9b repress frzb expression within the prechordal plate. frzb is required to protect the primary mouth precursor (oral placode) and the preplacodal ectoderm (PPE) from caudalizing signals, including Wnt signaling. (II) from 24hpf through 36hpf Wnt9b promotes D-V to D-I-V CNCC patterning at least partially through edn1. (III) From 36hpf to 48hpf, sox9a influences CNCC mesenchyme to differentiate into prechondrocytes, the jaw cartilage precursors. At this same time, pitx2 expression marks the inner primary mouth. (IV) Jaw cartilages begin anterior directed outgrowth from around the primary mouth. The lower face and jaws eventually form, comprising the secondary mouth. Outgrowth may be influenced by Wnt5b mediated convergent extension processes. Solid black arrows and bars indicate previously established genetic interactions. Orange arrows and bars indicate newly proposed genetic interactions. Dotted arrows and bars indicate speculative genetic interactions. 
Wnt9b is required in two phases: 1) D-I-V patterning of CNCC and 2) outgrowth towards the secondary mouth. Phase one includes CNCC patterning and possibly establishment of the primary mouth by dorsal anterior prechordal plate mesoderm (Figures 10(I)-(II)). Phase two includes jaw cartilage maturation and outgrowth, as well as repression of primary mouth markers (Figures 10(III)-(IV)). The detailed mechanisms underlying these phases need much additional work before they are fully understood. However, the current work serves to provide a broad overview of embryonic events that are crucial to formation of the oral-facial structures. Nevertheless, this work in total provides ample evidence that earlier patterning likely plays a larger role in craniofacial patterning and outgrowth than previously appreciated. If these results could be replicated in mammalian model systems, they would suggest a major reevaluation that would be necessary for judging the susceptibility timeline for these human embryonic disorders.

\section{Acknowledgements}

We thank the Nutrition Obesity Research Center Aquatic Animal Research Core, including Dr. Stephen Watts and Jeff Barry, for assistance in zebrafish husbandry and care. The NORC AARC is funded by NIH P30DK056336. We also thank Dr. Anil Challa for his expert technical advice.

\section{References}

[1] Strauss, R.P. (1999) The Organization and Delivery of Craniofacial Health Services: The State of the Art. Cleft Palate-Craniofacial Journal, 36, 189-195. http://dx.doi.org/10.1597/1545-1569(1999)036<0189:TOADOC>2.3.CO;2

[2] Schutte, B.C. and Murray, J.C. (1999) The Many Faces and Factors of Orofacial Clefts. Human Molecular Genetics, 8, 1853-1859. http://dx.doi.org/10.1093/hmg/8.10.1853

[3] Kamble, V.D., Parkhedkar, R.D., Sarin, S.P., Patil, P.G. and Kothari, B. (2013) Simplifying Cleft Surgery by Presurgical Nasoalveolar Molding (PNAM) for Infant Born with Unilateral Cleft Lip, Alveolus, and Palate: A Clinical Report. Journal of Prosthodontic Research, 57, 224-231. http://dx.doi.org/10.1016/j.jpor.2013.03.002

[4] Scapoli, L., Martinelli, M., Pezzetti, F., Carinci, F., Bodo, M., et al. (2002) Linkage Disequilibrium between GABRB 3 Gene and Nonsyndromic Familial Cleft Lip with or without Cleft Palate. Human Genetics, 110, 15-20. http://dx.doi.org/10.1007/s00439-001-0639-5

[5] Gaspar, D.A., Pavanello, R.C., Zatz, M., Passos-Bueno, M.R., Andre, M., et al. (1999) Role of the C677T Polymorphism at the MTHFR Gene on Risk to Nonsyndromic Cleft Lip with/without Cleft Palate: Results from a Case-Control Study in Brazil. American Journal of Medical Genetics, 87, 197-199. http://dx.doi.org/10.1002/(SICI)1096-8628(19991119)87:2<197::AID-AJMG15>3.0.CO;2-M

[6] van den Boogaard, M.J., Dorland, M., Beemer, F.A. and van Amstel, H.K. (2000) MSX1 Mutation Is Associated with Orofacial Clefting and Tooth Agenesis in Humans. Nature Genetics, 24, 342-343. http://dx.doi.org/10.1038/74155

[7] Wang, M.L., Pan, Y.C., Zhang, Z.D. and Wang, L. (2012) Three Polymorphisms in IRF6 and 8q24 Are Associated with Nonsyndromic Cleft Lip with or without Cleft Palate: Evidence from 20 Studies. American Journal of Medical Genetics Part A, 158A, 3080-3086.http://dx.doi.org/10.1002/ajmg.a.35634

[8] Jezewski, P.A., Vieira, A.R., Nishimura, C., Ludwig, B., Johnson, M., et al. (2003) Complete Sequencing Shows a Role for MSX1 in Non-Syndromic Cleft Lip and Palate. Journal of Medical Genetics, 40, 399-407. http://dx.doi.org/10.1136/jmg.40.6.399

[9] Kamamoto, M., Machida, J., Yamaguchi, S., Kimura, M., Ono, T., et al. (2011) Clinical and Functional Data Implicate the Arg(151)Ser Variant of MSX1 in Familial Hypodontia. European Journal of Human Genetics, 19, 844-850. http://dx.doi.org/10.1038/ejhg.2011.47

[10] Pauws, E., Peskett, E., Boissin, C., Hoshino, A., Mengrelis, K., et al. (2013) X-Linked CHARGE-Like Abruzzo-Erickson Syndrome and Classic Cleft Palate with Ankyloglossia Result from TBX22 Splicing Mutations. Clinical Genetics, 83, 352-358. http://dx.doi.org/10.1111/j.1399-0004.2012.01930.x

[11] Niemann, S., Zhao, C.F., Pascu, F., Stahl, U., Aulepp, U., et al. (2004) Homozygous Wnt3 Mutation Causes Tetra-Amelia in a Large Consanguineous Family. American Journal of Human Genetics, 74, 558-563. http://dx.doi.org/10.1086/382196

[12] Person, A.D., Beiraghi, S., Sieben, C.M., Hermanson, S., Neumann, A.N., et al. (2010) Wnt5a Mutations in Patients with Autosomal Dominant Robinow Syndrome. Developmental Dynamics, 239, 327-337.

[13] Beiraghi, S., Leon-Salazar, V., Larson, B.E., John, M.T., Cunningham, M.L., et al. (2011) Craniofacial and Intraoral Phenotype of Robinow Syndrome Forms. Clinical Genetics, 80, 15-24.

http://dx.doi.org/10.1111/j.1399-0004.2011.01683.x 
[14] Vieira, A.R., McHenry, T.G., Daack-Hirsch, S., Murray, J.C. and Marazita, M.L. (2008) A Genome Wide Linkage Scan for Cleft Lip and Palate and Dental Anomalies. American Journal of Medical Genetics Part A, 146A, 1406-1413. http://dx.doi.org/10.1002/ajmg.a.32295

[15] Maestri, N.E., Beaty, T.H., Hetmanski, J., Smith, E.A., McIntosh, I., et al. (1997) Application of Transmission Disequilibrium Tests to Nonsyndromic Oral Clefts: Including Candidate Genes and Environmental Exposures in the Models. American Journal of Medical Genetics, 73, 337-344.

http://dx.doi.org/10.1002/(SICI)1096-8628(19971219)73:3<337::AID-AJMG21>3.0.CO;2-J

[16] Shaw, G.M., Todoroff, K., Finnell, R.H., Rozen, R. and Lammer, E.J. (1999) Maternal Vitamin Use, Infant C677T Mutation in MTHFR, and Isolated Cleft Palate Risk. American Journal of Medical Genetics, 85, 84-85. http://dx.doi.org/10.1002/(SICI)1096-8628(19990702)85:1<84::AID-AJMG15>3.0.CO;2-V

[17] Natsume, N., Kawai, T., Ogi, N. and Yoshida, W. (2000) Maternal Risk Factors in Cleft Lip and Palate: Case Control Study. British Journal of Oral and Maxillofacial Surgery, 38, 23-25. http://dx.doi.org/10.1054/bjom.1999.0133

[18] Mostowska, A., Hozyasz, K.K., Biedziak, B., Wojcicki, P., Lianeri, M., et al. (2012) Genotype and Haplotype Analysis of WNT Genes in Non-Syndromic Cleft Lip with or without Cleft Palate. European Journal of Oral Sciences, 120, 1-8. http://dx.doi.org/10.1111/j.1600-0722.2011.00938.x

[19] Chiquet, B.T., Blanton, S.H., Bur,t A., Ma, D.Q., Stal, S., et al. (2008) Variation in Wnt Genes Is Associated with Non-Syndromic Cleft Lip with or without Cleft Palate. Human Molecular Genetics, 17, 2212-2218. http://dx.doi.org/10.1093/hmg/ddn121

[20] Hanken, J. and Thorogood, P. (1993) Evolution and Development of the Vertebrate Skull-The Role of Pattern-Formation. Trends in Ecology \& Evolution, 8, 9-15. http://dx.doi.org/10.1016/0169-5347(93)90124-8

[21] Thomson, K.S. (1993) The Skull: Patterns of Structural and Systematic Diversity. Vol. 2, In: Hanken, J. and Hall, B.K., Eds., Segmentation, the Adult Skull and the Problem of Homology, Chap. 2, University of Chicago Press, Chicago, 3668.

[22] Hildebrand, M. (1974) Analysis of Vertebrate Structure. Head Skeleton, Chap. 7, John Wiley \& Sons Inc., Hoboken, 125-155.

[23] Schilling, T.F. and Kimmel, C.B. (1997) Musculoskeletal Patterning in the Pharyngeal Segments of the Zebrafish Embryo. Development, 124, 2945-2960.

[24] Kimmel, C.B., Miller, C.T., Kruze, G., Ullmann, B., BreMiller, R.A., et al. (1998) The Shaping of Pharyngeal Cartilages during Early Development of the Zebrafish. Developmental Biology, 203, 245-263. http://dx.doi.org/10.1006/dbio.1998.9016

[25] Medeiros, D.M. and Crump, J.G. (2012) New Perspectives on Pharyngeal Dorsoventral Patterning in Development and Evolution of the Vertebrate Jaw. Developmental Biology, 371, 121-135. http://dx.doi.org/10.1016/j.ydbio.2012.08.026

[26] Saito-Diaz, K., Chen, T.W., Wang, X.X., Thorne, C.A., Wallace, H.A., et al. (2013) The Way Wnt Works: Components and Mechanism. Growth Factors, 31, 1-31. http://dx.doi.org/10.3109/08977194.2012.752737

[27] Song, L.Y., Li, Y.H., Wang, K., Wang, Y.-Z., Molotkov, A., et al. (2009) Lrp6-Mediated Canonical Wnt Signaling Is Required for Lip Formation and Fusion. Development, 136, 3161-3171. http://dx.doi.org/10.1242/dev.037440

[28] Witte, F., Bernatik, O., Kirchner, K., Masek, J., Mahl, A., et al. (2010) Negative Regulation of Wnt Signaling Mediated by $\mathrm{CK}_{1}$-Phosphorylated Dishevelled via Ror 2 . FASEB Journal, 24, 2417-2426. http://dx.doi.org/10.1096/fj.09-150615

[29] Kuhl, M., Geis, K., Sheldahl, L.C., Pukrop, T., Moon, R.T., et al. (2001) Antagonistic Regulation of Convergent Extension Movements in Xenopus by Wnt $/ \beta$-Catenin and Wnt/Ca ${ }^{2+}$ Signaling. Mechanisms of Development, 106, 61-76. http://dx.doi.org/10.1016/S0925-4773(01)00416-6

[30] Stoick-Cooper, C.L., Weidinger, G., Riehle, K.J., Hubbert, C., Major, M.B., et al. (2007) Distinct Wnt Signaling Pathways Have Opposing Roles in Appendage Regeneration. Development, 134, 479-489. http://dx.doi.org/10.1242/dev.001123

[31] Kikuchi, A., Yamamoto, H. and Sato, A. (2009) Selective Activation Mechanisms of Wnt Signaling Pathways. Trends in Cell Biology, 19, 119-129. http://dx.doi.org/10.1016/j.tcb.2009.01.003

[32] Sun, Y., Teng, I., Huo, R., Rosenfeld, M.G., Olson, L.E., et al. (2012) Asymmetric Requirement of Surface Epithelial $\beta$-Catenin during the Upper and Lower Jaw Development. Developmental Dynamics, 241, 663-674. http://dx.doi.org/10.1002/dvdy.23755

[33] Yamaguchi, T.P., Bradley, A., McMahon, A.P. and Jones, S. (1999) A Wnt5a Pathway Underlies Outgrowth of Multiple Structures in the Vertebrate Embryo. Development, 126, 1211-1223.

[34] He, F.L., Xiong, W., Yu, X.Y., Espinoza-Lewis, R., Liu, C., et al. (2008) Wnt5a Regulates Directional Cell Migration and Cell Proliferation via Ror2-Mediated Noncanonical Pathway in Mammalian Palate Development. Development, 
135, 3871-3879. http://dx.doi.org/10.1242/dev.025767

[35] Piotrowski, T., Schilling, T.F., Brand, M., Jiang, Y.J., Heisenberg, C.P., et al. (1996) Jaw and Branchial Arch Mutants in Zebrafish II: Anterior Arches and Cartilage Differentiation. Development, 123, 345-356.

[36] Lin, S.D., Baye, L.M., Westfall, T.A. and Slusarski, D.C. (2010) Wnt5b-Ryk Pathway Provides Directional Signals to Regulate Gastrulation Movement. Journal of Cell Biology, 190, 263-278. http://dx.doi.org/10.1083/jcb.200912128

[37] Juriloff, D.M., Harris, M.J., McMahon, A.P., Carroll, T.J. and Lidral, A.C. (2006) Wnt9b Is the Mutated Gene Involved in Multifactorial Nonsyndromic Cleft Lip with or without Cleft Palate in A/WySn Mice, as Confirmed by a Genetic Complementation Test. Birth Defects Research Part A: Clinical and Molecular Teratology, 76, 574-579. http://dx.doi.org/10.1002/bdra.20302

[38] Lan, Y., Ryan, R.C., Zhang, Z.Y., Bullard, S.A., Bush, J.O., et al. (2006) Expression of Wnt9b and Activation of Canonical Wnt Signaling during Midfacial Morphogenesis in Mice. Developmental Dynamics, 235, 1448-1454. http://dx.doi.org/10.1002/dvdy.20723

[39] Jin, Y.R., Han, X.H., Taketo, M.M. and Yoon, J.K. (2012) Wnt9b-Dependent FGF Signaling Is Crucial for Outgrowth of the Nasal and Maxillary Processes during Upper Jaw and Lip Development. Development, 139, 1821-1830. http://dx.doi.org/10.1242/dev.075796

[40] Ferretti, E., Li, B.S., Zewdu, R., Wells, V., Hebert, J.M., et al. (2011) A Conserved Pbx-Wnt-p63-Irf6 Regulatory Module Controls Face Morphogenesis by Promoting Epithelial Apoptosis. Developmental Cell, 21, 627-641. http://dx.doi.org/10.1016/j.devcel.2011.08.005

[41] Jezewski, P.A., Fang, P.K., Payne-Ferreira, T.L. and Yelick, P.C. (2008) Zebrafish Wnt9b Synteny and Expression during First and Second Arch, Heart, and Pectoral Fin Bud Morphogenesis. Zebrafish, 5, 169-177. http://dx.doi.org/10.1089/zeb.2007.0517

[42] Lu, F.I., Thisse, C. and Thisse, B. (2011) Identification and Mechanism of Regulation of the Zebrafish Dorsal Determinant. Proceedings of the National Academy of Sciences of the United States of America, 108, 15876-15880. http://dx.doi.org/10.1073/pnas.1106801108

[43] Cox, A.A., Jezewski, P.A., Fang, P.K. and Payne-Ferreira, T.L. (2010) Zebrafish Wnt9a, 9b Paralog Comparisons Suggest Ancestral Roles for Wnt9 in Neural, Oral-Pharyngeal Ectoderm and Mesendoderm. Gene Expression Patterns, 10, 251-258. http://dx.doi.org/10.1016/j.gep.2010.05.005

[44] Jezewski, P.A., Fang, P.K., Payne-Ferreira, T.L. and Yelick, P.C. (2009) Alternative Splicing, Phylogenetic Analysis, and Craniofacial Expression of Zebrafish tbx22. Developmental Dynamics, 238, 1605-1612. http://dx.doi.org/10.1002/dvdy.21962

[45] Miller, C.T., Schilling, T.F., Lee, K., Parker, J. and Kimmel, C.B. (2000) Sucker Encodes a Zebrafish Endothelin-1 Required for Ventral Pharyngeal Arch Development. Development, 127, 3815-3828.

[46] Clouthier, D.E., Garcia, E. and Schilling, T.F. (2010) Regulation of Facial Morphogenesis by Endothelin Signaling: Insights from Mice and Fish. American Journal of Medical Genetics Part A, 152A, 2962-2973. http://dx.doi.org/10.1002/ajmg.a.33568

[47] Compagnucci, C., Debias-Thiabaud, M., Coolen, M., Fish, J., Griffin, J.N., Bertocchini, F., Minoux, M., Rijli, F.M., Borday-Birraux, V., Casane, D., Sylvie, M. and Depew, M.J. (2013) Pattern and Polarity in the Development and Evolution of the Gnathostome Jaw: Both Conservation and Heterotopy in the Branchial Arches of the Shark, Scyliorhinuscanicula. Developmental Biology, 377, 428-448. http://dx.doi.org/10.1016/j.ydbio.2013.02.022

[48] Talbot, J.C., Johnson, S.L. and Kimmel, C.B. (2010) hand2 and Dlx Genes Specify Dorsal, Intermediate and Ventral Domains within Zebrafish Pharyngeal Arches. Development, 137, 2507-2517. http://dx.doi.org/10.1242/dev.049700

[49] Miller, C.T., Swartz, M.E., Khuu, P.A., Walker, M.B., Eberhart, J.K., et al. (2007) mef2ca Is Required in Cranial Neural Crest to Effect Endothelin1 Signaling in Zebrafish. Developmental Biology, 308, 144-157. http://dx.doi.org/10.1016/j.ydbio.2007.05.018

[50] Walker, M.B., Miller, C.T., Coffin Talbot, J., Stock, D.W. and Kimmel, C.B. (2006) Zebrafish Furin Mutants Reveal Intricacies in Regulating Endothelin1 Signaling in Craniofacial Patterning. Developmental Biology, 295, $194-205$. http://dx.doi.org/10.1016/j.ydbio.2006.03.028

[51] Zuniga, E., Rippen, M., Alexander, C., Schilling, T.F. and Crump, J.G. (2011) Gremlin 2 Regulates Distinct Roles of BMP and Endothelin 1 Signaling in Dorsoventral Patterning of the Facial Skeleton. Development, 138, 5147-5156. http://dx.doi.org/10.1242/dev.067785

[52] Zuniga, E., Stellabotte, F. and Crump, J.G. (2010) Jagged-Notch Signaling Ensures Dorsal Skeletal Identity in the Vertebrate Face. Development, 137, 1843-1852. http://dx.doi.org/10.1242/dev.049056

[53] Depew, M.J., Lufkin, T. and Rubenstein, J.L. (2002) Specification of Jaw Subdivisions by Dlx Genes. Science, 298, 381-385. http://dx.doi.org/10.1126/science.1075703 
[54] Sperber, S.M., Saxena, V., Hatch, G. and Ekker, M. (2008) Zebrafish $d l x 2 a$ Contributes to Hindbrain Neural Crest Survival, Is Necessary for Differentiation of Sensory Ganglia and Functions with dlx1a in Maturation of the Arch Cartilage Elements. Developmental Biology, 314, 59-70. http://dx.doi.org/10.1016/j.ydbio.2007.11.005

[55] Qiu, M., Bulfone, A., Martinez, S., Meneses, J.J., Shimamura, K., et al. (1995) Null Mutation of Dlx-2 Results in abnormal Morphogenesis of Proximal First and Second Branchial Arch Derivatives and Abnormal Differentiation in the Forebrain. Genes Development, 9, 2523-2538. http://dx.doi.org/10.1101/gad.9.20.2523

[56] Jeong, J., Cesario, J., Zhao, Y.G., Burns, L., Westphal, H., et al. (2012) Cleft Palate Defect of Dlx1/2-/- Mutant Mice Is Caused by Lack of Vertical Outgrowth in the Posterior Palate. Developmental Dynamics, 241, 1757-1769. http://dx.doi.org/10.1002/dvdy.23867

[57] Soukup, V., Horacek, I. and Cerny, R. (2013) Development and Evolution of the Vertebrate Primary Mouth. Journal of Anatomy, 222, 79-99. http://dx.doi.org/10.1111/j.1469-7580.2012.01540.x

[58] Dickinson, A. and Sive, H. (2007) Positioning the Extreme Anterior in Xenopus: Cement Gland, Primary Mouth and Anterior Pituitary. Seminars in Cell Developmental Biology, 18, 525-533. http://dx.doi.org/10.1016/j.semcdb.2007.04.002

[59] Bhat, N., Kwon, H.J. and Riley, B.B. (2013) A Gene Network That Coordinates Preplacodal Competence and Neural Crest Specification in Zebrafish. Developmental Biology, 373, 107-117. http://dx.doi.org/10.1016/j.ydbio.2012.10.012

[60] Kwon, H.J., Bhat, N., Sweet, E.M., Cornell, R.A. and Riley, B.B. (2010) Identification of Early Requirements for Preplacodal Ectoderm and Sensory Organ Development. PLoS Genetics, 6, e1001133. http://dx.doi.org/10.1371/journal.pgen.1001133

[61] Harden, M.V., Pereiro, L., Ramialison, M., Wittbrodt, J., Prasad, M.K., et al. (2012) Close Association of Olfactory Placode Precursors and Cranial Neural Crest Cells Does Not Predestine Cell Mixing. Developmental Dynamics, 241, 1143-1154. http://dx.doi.org/10.1002/dvdy.23797

[62] Christophorou, N.A.D., Bailey, A.P., Hanson, S. and Streit, A. (2009) Activation of Six1 Target Genes Is Required for Sensory Placode Formation. Developmental Biology, 336, 327-336. http://dx.doi.org/10.1016/j.ydbio.2009.09.025

[63] Ishihara, T., Ikeda, K., Sato, S., Yajima, H. and Kawakami, K. (2008) Differential Expression of Eya1 and Eya2 during Chick Early Embryonic Development. Gene Expression Patterns, 8, 357-367. http://dx.doi.org/10.1016/j.gep.2008.01.003

[64] Dickinson, A.J. and Sive, H.L. (2009) The Wnt Antagonists Frzb-1 and Crescent Locally Regulate Basement Membrane Dissolution in the Developing Primary Mouth. Development, 136, 1071-1081. http://dx.doi.org/10.1242/dev.032912

[65] Pillai, K.G., Kamath, V.V., Kumar, G.S. and Nagamani, N. (1990) Persistent Buccopharyngeal Membrane with Cleft-Palate-A Case-Report. Oral Surgery, Oral Medicine, Oral Pathology, 69, 164-166. http://dx.doi.org/10.1016/0030-4220(90)90319-N

[66] Watts, S.A., Powell, M. and D’Abramo, L.R. (2012) Fundamental Approaches to the Study of Zebrafish Nutrition. ILAR Journal, 53, 144-160. http://dx.doi.org/10.1093/ilar.53.2.144

[67] Bill, B.R., Petzold, A.M., Clark, K.J., Schimmenti, L.A. and Ekker, S.C. (2009) A Primer for Morpholino Use in Zebrafish. Zebrafish, 6, 69-77. http://dx.doi.org/10.1089/zeb.2008.0555

[68] Thisse, C. and Thisse, B. (2008) High-Resolution in Situ Hybridization to Whole-Mount Zebrafish Embryos. Nature Protocols, 3, 59-69. http://dx.doi.org/10.1038/nprot.2007.514

[69] Shwartz, Y., Farkas, Z., Stern, T., Aszodi, A. and Zelzer, E. (2012) Muscle Contraction Controls Skeletal Morphogenesis through Regulation of Chondrocyte Convergent Extension. Developmental Biology, 370, 154-163. http://dx.doi.org/10.1016/j.ydbio.2012.07.026

[70] O’Brien, E.K., d’Alencon, C., Bonde, G., Li, W., Schoenebeck, J., et al. (2004) Transcription Factor Ap-2 $\alpha$ Is Necessary for Development of Embryonic Melanophores, Autonomic Neurons and Pharyngeal Skeleton in Zebrafish. Developmental Biology, 265, 246-261. http://dx.doi.org/10.1016/j.ydbio.2003.09.029

[71] Knight, R.D., Javidan, Y., Zhang, T.L., Nelson, S. and Schilling, T.F. (2005) AP2-Dependent Signals from the Ectoderm Regulate Craniofacial Development in the Zebrafish Embryo. Development, 132, 3127-3138. http://dx.doi.org/10.1242/dev.01879

[72] Yan, Y.L., Willoughby, J., Liu, D., Crump, J.G., Wilson, C., et al. (2005) A Pair of Sox: Distinct and Overlapping Functions of Zebrafish Sox9 Co-Orthologs in Craniofacial and Pectoral Fin Development. Development, 132: 10691083. http://dx.doi.org/10.1242/dev.01674

[73] Curtin, E., Hickey, G., Kamel, G., Davidson, A.J. and Liao, E.C. (2011) Zebrafish Wnt9a Is Expressed in Pharyngeal Ectoderm and Is Required for Palate and Lower Jaw Development. Mechanisms of Development, 128, 104-115. http://dx.doi.org/10.1016/j.mod.2010.11.003 
[74] Liu, Y. and Semina, E.V. (2012) pitx2 Deficiency Results in Abnormal Ocular and Craniofacial Development in Zebrafish. PLoS ONE, 7, e30896. http://dx.doi.org/10.1371/journal.pone.0030896

[75] Alexander, C., Zuniga, E., Blitz, I.L., Wada, N., Le Pabic, P., et al. (2011) Combinatorial Roles for BMPs and Endothelin 1 in Patterning the Dorsal-Ventral Axis of the Craniofacial Skeleton. Development, 138, 5135-5146. http://dx.doi.org/10.1242/dev.067801

[76] Phillips, B.T., Kwon, H.J., Melton, C., Houghtaling, P., Fritz, A., et al. (2006) Zebrafish msxB, msxC and msxE Function Together to Refine the Neural-Nonneural Border and Regulate Cranial Placodes and Neural Crest Development. Developmental Biology, 294, 376-390. http://dx.doi.org/10.1016/j.ydbio.2006.03.001

[77] Finnerty, J.R., Mazza, M.E. and Jezewski, P.A. (2009) Domain Duplication, Divergence, and Loss Events in Vertebrate Msx Paralogs Reveal Phylogenomically Informed Disease Markers. BMC Evolutionary Biology, 9, 18.

[78] Weinberg, S.M., Naidoo, S.D., Bardi, K.M., Brandon, C.A., Neiswanger, K., et al. (2009) Face Shape of Unaffected Parents with Cleft Affected Offspring: Combining Three-Dimensional Surface Imaging and Geometric Morphometrics. Orthodontics Craniofacial Research, 12, 271-281. http://dx.doi.org/10.1111/j.1601-6343.2009.01462.x

[79] Gieseler, K., Graba, Y., Mariol, M.C., Wilder, E.L., Martinez-Arias, A., et al. (1999) Antagonist Activity of DWnt-4 and Wingless in the Drosophila Embryonic Ventral Ectoderm and in Heterologous Xenopus Assays. Mechanisms of Development, 85, 123-131. http://dx.doi.org/10.1016/S0925-4773(99)00097-0 
(a)

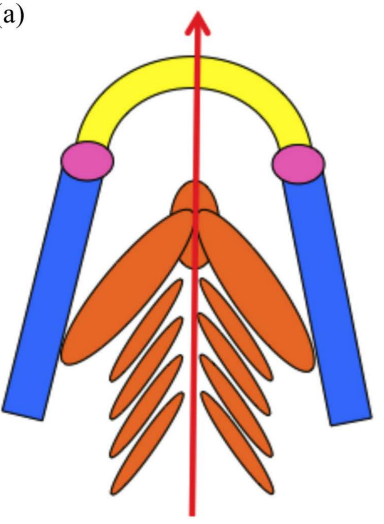

(b)

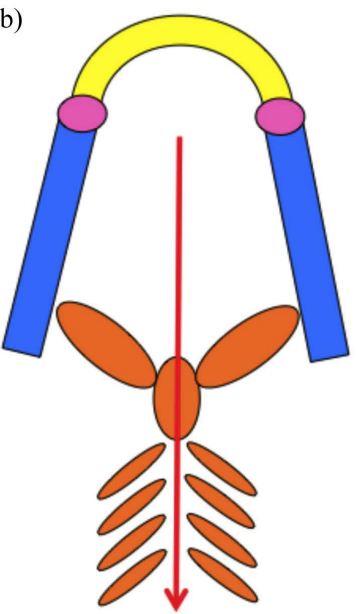

(f)

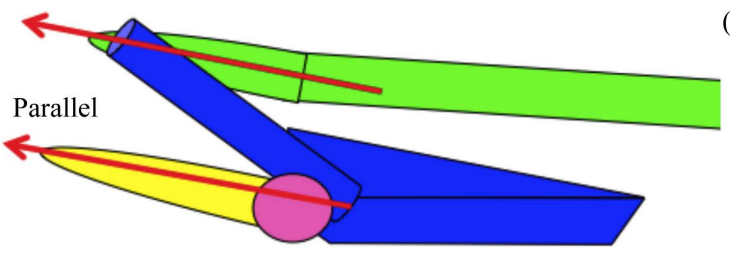

(c)

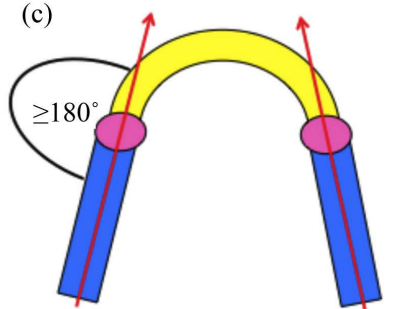

(d)

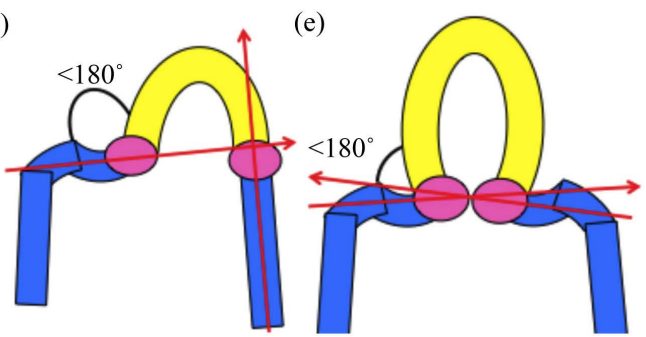

(g)

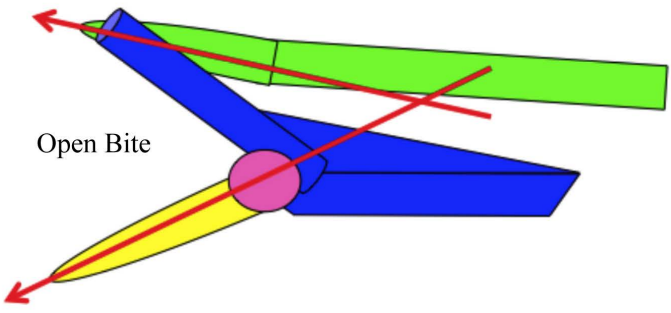

Supplemental Figure 1. Embryos were scored for common phenotypes when viewed in either a ventral or lateral view. Each of the landmarked phenotypes below were scored as a 0 (unaffected) or 1 (affected). In a Ventral view (panels (a)-(e), points were awarded to any embryo that exhibited the following phenotype changes: 1) Inverted Ceratohyal orientation, such that while normal paired ceratohyals (large brown ovals) point anteriorly (a), the morphants frequently had ceratohyals pointed posteriorly (b). One point. 2) A Meckel's-Palatoquadrate Joint (MPJ) angle of $<180^{\circ}$. Meckel's (yellow) and palatoquadrate cartilages (blue). One point was scored for each side that had a MPJ of $<180^{\circ}$. Normal MPJ angles were always $180^{\circ}$ (c) and morphants frequently exhibited less than $180^{\circ}$ joint angles. The Meckel's and Palatoquadrate were frequently fused whenever the MPJ was $<180^{\circ}$. One point for unilateral (d), two points for bilateral (e) MPJoints $<180^{\circ}$. In lateral views, (panels (f), (g), embryos were awarded one point whenever they exhibited: 3) An open bite. Normal embryos had their Meckel's cartilage and the anterior ethmoid plate in a parallel orientation, while morphants had Meckel's cartilages distending ventrally from the ethmoid. One point. Severe embryos were defined as those with three or four points. Moderate embryos were defined as those with two points, mild embryos only had one point, and normal embryos had zero points. 


\section{(a)} wnt9b
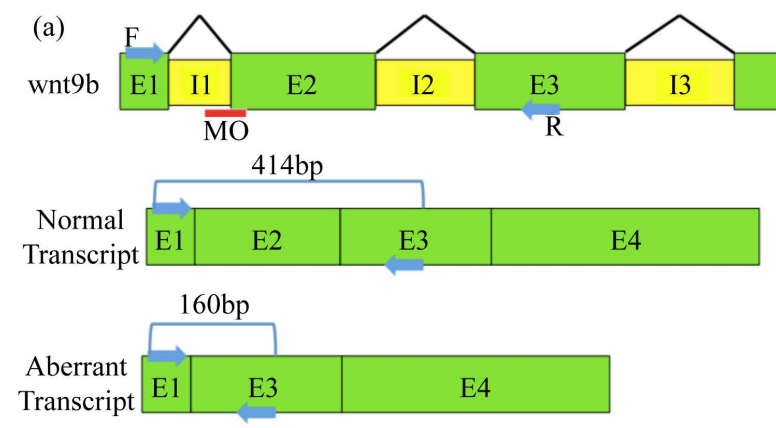

(c) $100 \%$ E4
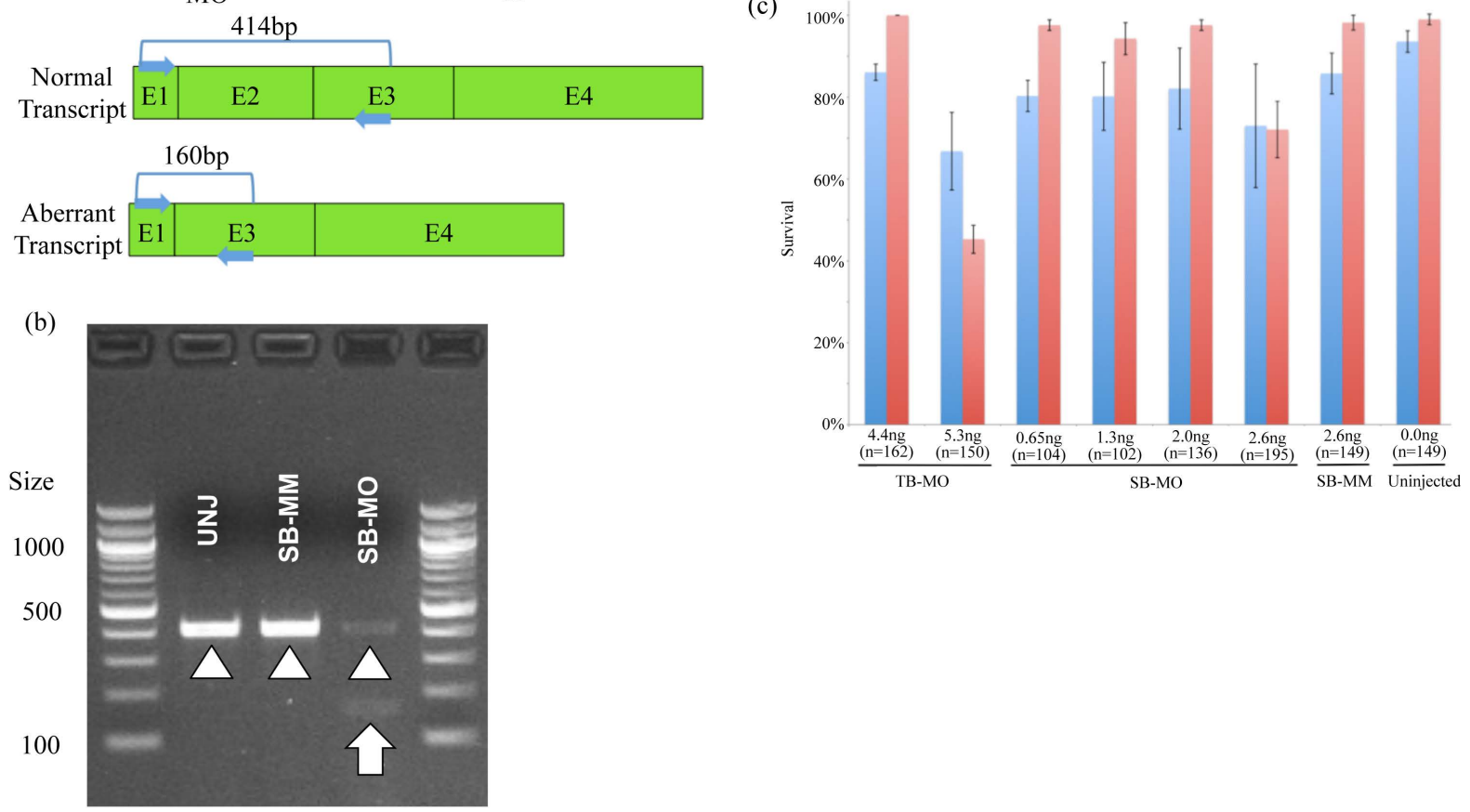

Supplemental Figure 2. Wnt9b morpholino knockdown validation. (a) Wnt9b Transcript Targeting Strategy. (a) The Wnt9b coding region consists of 4 exons and three introns. A splice blocking morpholino (SB-MO) was designed to target the intron-exon border of intron 1 and exon 2 (red bar, MO). Primer locations for diagnostic RT-PCR are indicated as blue arrows. In addition we designed a translation blocking morpholino targeting the Wnt9b start codon. (B) RT-PCR from uninjected control (UNJ), mismatch control injected (SB-MM, 2.6 ng), or splice blocking morpholino injected (SB-MO, 2.6 ng) cDNA preps. White arrowheads indicate the normal 414 bp product from a normal Wnt9b transcript. White arrows indicate the aberrant 160bp product from a transcript with exon 2 deleted. Note that in control groups only the normal product is present, and in the SB-MO group a normal and aberrant product are present. Sequencing of the 160 bp product confirmed it was an aberrant Wnt9b transcript with exon 2 deleted. (c) Survival of morpholino injected embryos from 0 - 1dpf (blue bars) and from 1 - 5dpf (red bars). The amount of morpholino injected is indicated in ng. The total number (n) of embryos injected and followed from 0 - 1dpf and 1 - 5dpf is indicated. Error bars are standard error of means determined from 3 biological replicates each.

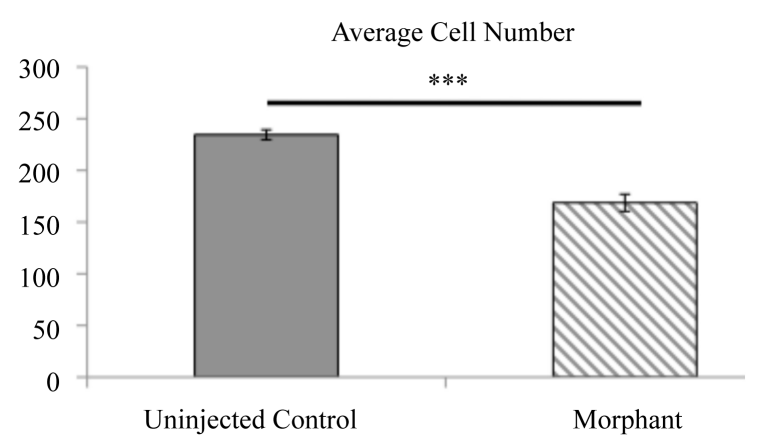

(a)

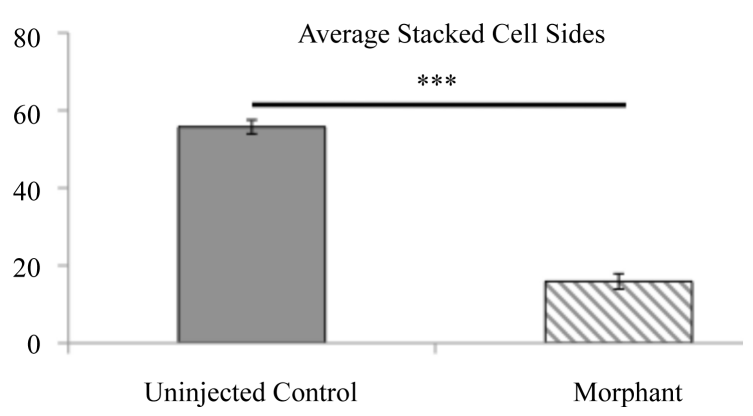

(b)

Supplemental Figure 3. Wnt9b morphant PQs have disrupted cellular orientation. Uninjected control and TB-MO injected embryo PQs were dissected out and total cell number (a) and cell stacking (b) were quantified. Morphants have approximately $30 \%$ reduced cell number and approximately $70 \%$ reduced cell stacking. Uninjected $n=4$, morphants $n=10$. Significance was calculated using a T-test, ${ }^{* * *} \mathrm{p}<0.001$. 


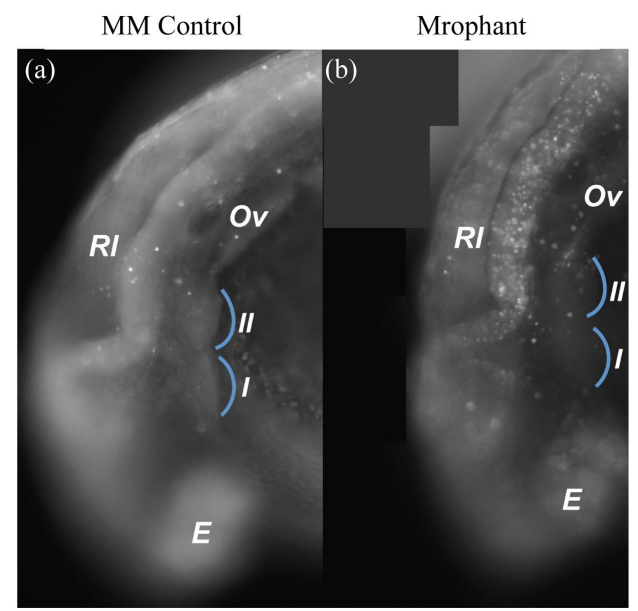

Supplemental Figure 4. Wnt9b morphants do not display increased apoptosis in the first pharyngeal arch. (a) (b) Lateral Views. (a) MM control and (b) morphant embryos were aged to 26hpf and subjected to acridine orange staining to assay cell death. Blue arcs indicate PA1\&2. Note that apoptotic cells within the PAs are not increased in morphants. Apoptotic cells within dorsal neural structures are increased in morphants. $\mathrm{Br}=$ brain, $E=$ eye, $\mathrm{Ov}$ = otic vesicle, $R l=$ rhombic lips.
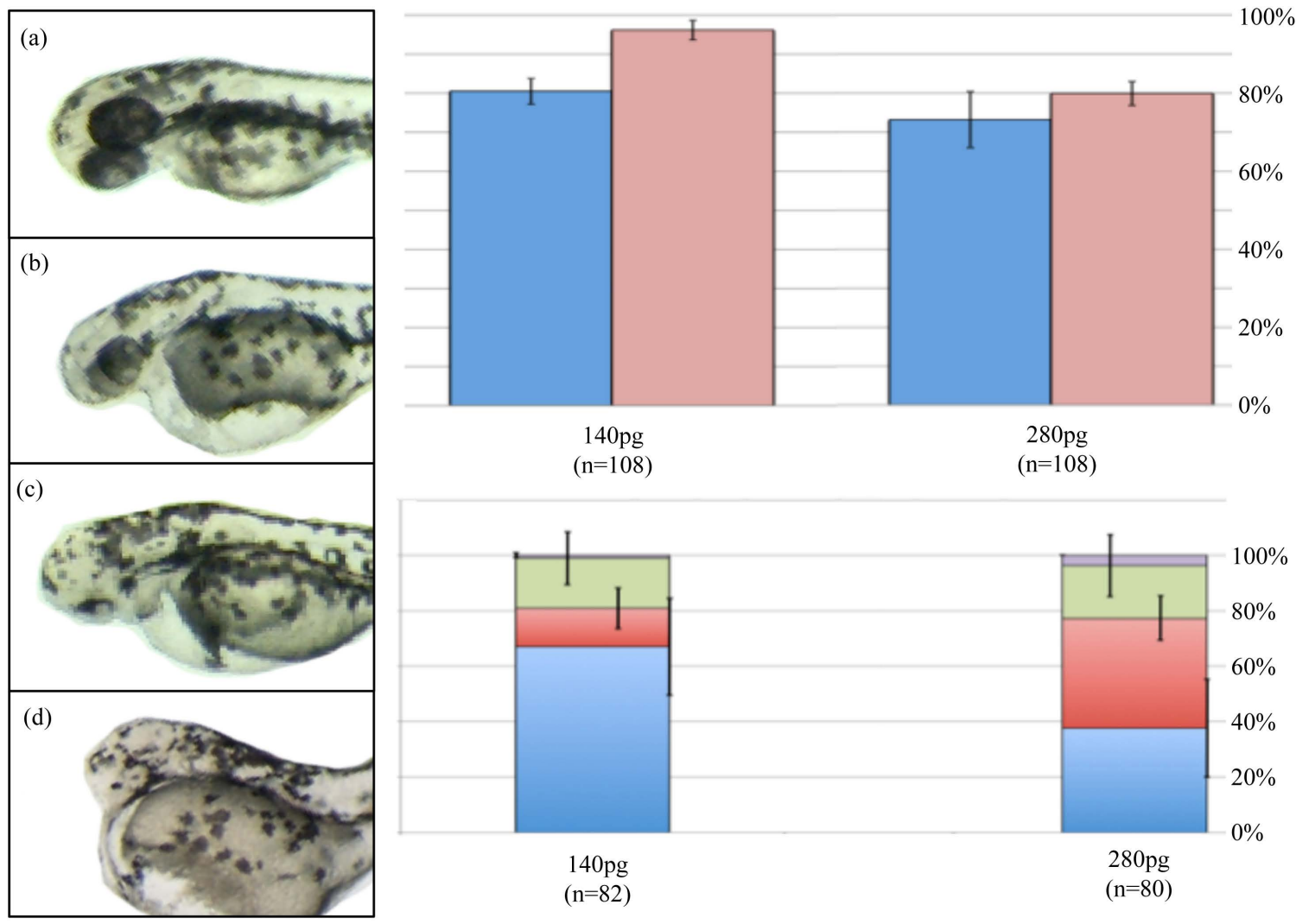

$\stackrel{\pi}{2}$

Supplemental Figure 5. Wnt9b overexpression reduces dorsal/neural tissues. (a)-(d) 1-cell aged embryos were injected with either $140 \mathrm{pg}$ or $280 \mathrm{pg}$ of synthetic Wnt9b mRNA and monitored for survival (e) and 48hpf eye phenotypes (f). Live embryos scored for (a) normal eyes, (b) small eyes, (c) cyclopia, and (d) no eye phenotypes that are represented in (f). (b) Blue columns are the 0 - 1dpf survival, red columns are the 1 - 2dpf survival. (f) Distribution of eye phenotypes of Wnt9b overexpressing embryos. Purple $=$ no eyes, green $=$ cyclopic, red $=$ small eyes, blue $=$ normal eyes. 